\title{
Elucidation of Excitation Energy Dependent Correlated Triplet Pair Formation Pathways in an Endothermic Singlet Fission System
}

Arya Thampi ${ }^{1}$, Hannah L. Stern ${ }^{1}$, Alexandre Cheminal ${ }^{1}$, Murad J. Y. Tayebjee ${ }^{1}$, Anthony J. Petty $\mathrm{II}^{2}$, John E. Anthony ${ }^{2}$ and Akshay Rao* ${ }^{1}$

${ }^{1}$ Cavendish Laboratory, University of Cambridge, UK.

${ }^{2}$ University of Kentucky, Lexington, USA. 


\begin{abstract}
Singlet fission is the spin allowed conversion of a photo-generated singlet exciton into two triplet excitons in organic semiconductors, which could enable single-junction photovoltaic cells to break the Shockley-Queisser limit. The conversion of singlets to free triplets is mediated by an intermediate correlated triplet pair (TT) state, but an understanding of how the formation and dissociation of these states depend on energetics and morphology is lacking. In this study, we probe the dynamics of TT states in a model endothermic fission system, TIPS-Tc nanoparticles, which show a mixture of crystalline and disordered regions. We observe the formation of different TT states, with varying yield and different rates of singlet decay, depending on the excitation energy. An emissive TT state is observed to grow in over 1 ns when exciting at $480 \mathrm{~nm}$, in contrast to excitation at lower energies where this emissive TT state is not observed. This suggests that the pathway for singlet fission in these nanoparticles is strongly influenced by the initial sub-100 fs relaxation of the photoexcited state away from the Frank-Condon point, with multiple possible TT states. On nanosecond timescales, the TT states are converted to free triplets, which suggests that TT states might diffuse into the disordered regions of the nanoparticles where their breakup to free triplets is favored. The free triplets then decay on $\mu \mathrm{s}$ timescales, despite the confined nature of the system. Our results provide important insights into the mechanism of endothermic singlet fission and the design of nanostructures to harness singlet fission.
\end{abstract}




\section{Introduction}

Conventional single junction photovoltaic (PV) devices are limited to an energy conversion efficiency of $33.7 \%$ under 1 Sun illumination ${ }^{1}$. These losses arise largely from transmission of sub-band gap photons and the thermalisation of photon energy in excess of the band gap. Singlet exciton fission is a process wherein an optically generated spin-0 singlet exciton splits to form two spin-1 triplet excitons. The process is mediated by triplet-pair state (TT state), which is generated from the photogenerated singlet state and in which the triplets initially have (anti-)correlated spins $^{2,3}$, i.e. a spin- 0 state. The generation of two excitons from a single photon can be exploited to augment the current of solar cell, raising the thermodynamic limiting efficiency to $45.9 \%^{4-8}$.

Singlet fission is observed in various organic materials such as acenes ${ }^{9-12}$, biradicaloids ${ }^{13,14}$, polymer aggregates such as carotenoids ${ }^{15-17}$ etc. Conventionally, the process of singlet fission is contingent on the condition that the energy of the singlet state must be close to twice the energy of the triplet state. The difference between the singlet energy and twice that of the triplet energy, $\Delta \mathrm{E}=2 \mathrm{E}\left(\mathrm{T}_{1}\right)-\mathrm{E}\left(\mathrm{S}_{1}\right)$, is used to define singlet fission materials into exothermic $(\Delta \mathrm{E}<0)$, isoergic $(\Delta \mathrm{E}=0)$ and endothermic $(\Delta \mathrm{E}>0)$ systems. While singlet fission has been extensively studied in exothermic systems, endothermic systems are of particular interest due to their potential use in devices (see below). But these systems are not as widely studied and not as well understood as exothermic fission systems. This is true even for Tetracene, the most extensively studied endothermic singlet fission system ${ }^{18-22}$.

Here, we study a tetracene derivative 5,12- bis(triisopropylsilylethynyl)tetracene (TIPS-Tc). TIPS-Tc is more soluble in organic solvents, improving its processing from solution and opening up interesting device fabrication opportunities. Importantly, the triplet energy of TIPS-Tc, $1.2-1.3 \mathrm{eV}$ means that it could compliment Si in PV devices (bandgap $1.1 \mathrm{eV}$ ). This makes it an interesting system to probe the dynamics of endothermic fission. The various excited states in the process of singlet fission and their absorption signatures have been previously characterized for solution ${ }^{23}$ and films ${ }^{24}$ of TIPS- Tc. Recently, for TIPS- Tc, it has been observed 
that TT states are formed from the photogenerated singlet state on $300 \mathrm{fs}$ timescales, mush faster than previously considered possible in endothermic fission systems. The subsequent behavior of these TT states was found to be dependent on morphology. In crystalline films free triplet excitons are not formed and TT states remain bound, while free triplets are formed on ns timescales via the breakup of TT states in disordered films ${ }^{24}$. This difference has been attributed to the change in triplet hopping rates between crystalline and disordered regions. These surprising results raise the questions of how the formation and decay dynamics of TT states in endothermic fission systems are dependent on nano- morphology, something that has not been probed till date. This is in contrast to say the field of organic photovoltaics where the critical effect of nano- morphology on photophysics and device performance has been widely recognized and extensive studies have been carried out ${ }^{25-33}$. Thus, if endothermic fission materials are to be used in practical devices, it is important to establish the link between nano- morphology and photophysics, with the aim of providing design rules for future materials and devices.

In this work, we study the formation and decay dynamics of TT states in a model singlet fission system, nanoparticles of TIPS-Tc. Nanoparticles of acene derivatives of pentacene and tetracene have been studied ${ }^{34-36}$ previously in nanoparticles of size above $100 \mathrm{~nm}$, but the dynamics of TT states have not been explicitly addressed.-As we discuss below, nanoparticles in this study show a mixture of crystalline and disordered regions. This is unlike the model crystalline and disordered films studied previously. It practical devices, it is likely that organic thin films will show a mixture of crystalline and disordered regions, but studying such films is complicated by problems of sample reproducibility. In contrast, the nanoparticle system used here allows us to form reproducible nanostructures consisting of a crystalline core surrounded by a disordered shell. The size of the nanoparticles is also controlled to be comparable to the exciton diffusion length in these systems. Using ultrafast pumpprobe spectroscopy and time-resolved photoluminescence spectroscopy, we identify the spectral features associated with the TT state and observe its formation in sub-500 fs timescales. We observe that the formation of this state is highly dependent on the excitation energy; higher photon energy leads to slower formation of TT and slower decay of the stimulated emission from the singlet exciton, as well as lower overall TT 
state yield. Remarkably, we observe the formation of emissive TT states capable of stimulated emission, for certain pump energies. On nanosecond timescales, we observe the dissociation of the TT state to form free triplets that live for several microseconds, even within the confined geometry of the nanoparticle. Our results suggest that the dynamics of the TT state are highly morphology dependent and show that the relaxation of the initially formed TT states can occur via multiple pathways, leading to very different relaxed TT states at longer times. These results provide a crucial window into how nano- morphology and energetics effect TT state dynamics and thus control the singlet fission process.

\section{Materials and Methods}

\section{Materials}

5,12- bis(triisopropylsilylethynyl)tetracene or TIPS-Tc, was synthesized as described previously $^{37}$. Reagent grade Tetrahydrofuran (THF), deuterated THF (THF- d8), $\mathrm{D}_{2} \mathrm{O}$, Gelatin type A polymer from porcine skin were purchased from Sigma-Aldrich and were used without further purification except for gelatin. While preparing gelatin for making thin films of the sample, the high molecular weight component of the polymer sample was separated by centrifugation and was used after washing with acetone.

\section{Sample preparation}

Nanoparticles of the acene were synthesized using an extensively reported method of re-precipitation ${ }^{34-36,38,39}$, starting from a batch of crystalline powder of TIPS- Tc. 100 $\mu \mathrm{L}$ of a $0.6 \mathrm{mg} / \mathrm{mL}$ solution of the acene in tetrahydrofuran (THF) was rapidly injected into $5 \mathrm{~mL}$ of de-ionized water under stirring and left for growth at room temperature in a dark, inert environment. After 30 minutes of stirring of the solution steady- state absorption and photoluminescence measurements were performed. After the reaction, the nanoparticle solution and spincoated films were observed to degrade over time and upon laser exposure. To improve the stability of the nanoparticles for spectroscopic studies, the nanoparticles were embedded in a matrix made of gelatin polymer. For this, the high molecular part of type A gelatin was obtained by 
dissolving in water at $55-60{ }^{\circ} \mathrm{C}$ and precipitating it out by centrifugation. $200 \mu \mathrm{L}$ of the nanoparticles dispersed in water was then added to the polymer solution slowly, under stirring and maintaining the temperature at $55^{\circ} \mathrm{C}$ for 30 minutes. To make films of the sample, $150 \mu \mathrm{L}$ of the nanoparticle- gelatin solution were drop- cast on spectrosil substrates in an inert atmosphere at room temperature for the gelatin to solidify. The films were encapsulated in inert atmosphere and used for spectroscopic studies after several days. For transmission electron microscopy (TEM), $20 \mu \mathrm{L}$ of the nanoparticles dispersed in water was drop- cast on a carbon film coated copper TEM grid with a mesh size of 200 .

\section{Characterization}

UV-Vis absorption studies of all the samples were recorded using a Cary 400 UVVisible spectrometer in the wavelength range of $350-800 \mathrm{~nm}$, in a $10 \mathrm{~mm}$ path length quartz cuvette. Transmission electron microscopy (TEM) measurements of the samples were done on a Fei Tecnai F20 200 kV high-resolution electron microscope. Analysis of TEM images were done using Image $1.50 \mathrm{~b}$ and simulation of $\mathrm{X}$ - ray diffraction pattern from the crystal structure was performed by using Mercury 3.9 (build RC1).

Steady- state photoluminescence spectra of the samples were recorded with a pulsed laser (PicoQuant LDH400 $40 \mathrm{MHz}$ ) at $470 \mathrm{~nm}$ and collected on a $500 \mathrm{~mm}$ focal length spectrograph (Princeton Instruments, SpectraPro2500i) with a cooled CCD camera. To record the time- resolved emission scan (TRES) or photoluminescence decay of the samples, time-correlated single photon counting (TCSPC) was performed, which is incorporated with the above-mentioned steady- state setup, with a time resolution of $300 \mathrm{ps}$.

The TA experiments conducted on gelatin matrix samples in different temporal and spectral time domains: ultrafast time delay (40 fs - $180 \mathrm{ps}$ ), short time delay (500 fs $1.2 \mathrm{~ns})$ and electronically controlled long time delay ( $1 \mathrm{~ns}-1 \mathrm{~ms})$ experiments using visible $(520 \mathrm{~nm}-850 \mathrm{~nm})$ and near- IR $(920 \mathrm{~nm}-1200 \mathrm{~nm})$ probes. The ultra-fast TA experiments were performed using a Yb-based amplified system (PHAROS, Light Conversion) providing $14.5 \mathrm{~W}$ at $1025 \mathrm{~nm}$ and $38 \mathrm{kHz}$ repetition rate. The probe 
beam is generated by focusing a portion of the fundamental in a $4 \mathrm{~mm}$ YAG substrate, and spans from 520- $900 \mathrm{~nm}$. The pump beam is generated in a non-collinear optical parametric amplifiers (NOPAs; $37^{\circ}$ cut BBO, type I, $5^{\circ}$ external angle) pumped with the third harmonic of the source. The NOPA outputs $1 \mathrm{~mW}$ power centered at $530 \mathrm{~nm}$, with pulse durations $<12$ fs (upper limit determined by frequency resolved opticalgating, FROG). The white light is delayed using a computer-controlled piezoelectric translation stage, and a sequence of probe pulses with and without pump is generated using a chopper wheel on the pump beam. After the sample, the probe pulses are seeded through a visible (550 $\mathrm{nm}$ blazed grating) spectrograph, and imaged using an InGaAs photodiode array camera (Sensors Unlimited).

For short time measurements ( $1 \mathrm{ps}-1.2 \mathrm{~ns})$, the laser source that is used to generate the probe and pump (short time delay) beams with a frequency of $1 \mathrm{kHz}$ was a Ti: sapphire amplifier system (Spectra- Physics Solstice). A part of the $1 \mathrm{kHz}$ pulse pumps a TOPAS optical parametric amplifier whose output is led to a delay stage that helps in producing a tunable excitation source for short-time measurements. Another part of the output from the Ti: sapphire system is used to generate broadband probe beams in the visible and near- IR region. Excitation energy dependence $\left(\lambda_{\mathrm{exc}}=532\right.$ $\mathrm{nm}, 500 \mathrm{~nm}$ and $480 \mathrm{~nm}$ ) and fluence dependence measurements were also carried out on the same sample with the above-mentioned conditions. For long time delay measurements (1 ns - $100 \mu \mathrm{s})$ an Nd: YVO4 laser (AOT-YVO-25QSPX, Advanced Optical Technologies) with electronically controlled delay was used. The pump and probe beams are overlapped on the sample adjacent to a reference probe beam. This reference is used to account for any shot-to- shot variation in transmission. The beams are focused into an imaging spectrometer (Andor, Shamrock SR 303i) and detected using a pair of linear image sensors (Hamamatsu, G11608) driven and read out at the full laser repetition rate by a custom-built board from Stresing Entwicklungsburo. A mechanical/electronic chopper is used to have pump on and pump off periods during all measurements, that enable the system software to calculate differential transmission, $\Delta \mathrm{T} / \mathrm{T}$.

In pump-probe experiments as described above, differential transmission $(\Delta T / T)$ obtained in the presence and absence of the pump pulse shows features that define 
excited states in the TA spectra. The positive features represent ground state bleach (GSB) and stimulated emission (SE) of the singlet state in ground and excited state configurations $\left(\mathrm{S}_{0}\right.$ and $\left.\mathrm{S}_{1}\right)$. The negative features denote the process of photo-induced absorption (PIA) of different excited state species such as singlets $\left(\mathrm{S}_{1}\right)$, correlated triplet pairs (TT), free triplets $\left(\mathrm{T}_{1}\right)$ etc. To identify multiple species from the transient absorption data thus collected, decay- associated spectral (DAS) analysis that uses single value decomposition (SVD) was done. A global population fit by means of a genetic algorithm was also used in identifying distinct spectral species and extracting respective kinetics.

\section{Results and Discussion}

The nanoparticles of TIPS- Tc were embedded in a gelatin matrix as explained in the experimental section, and were used for all the measurements presented in this study after several days of ageing.

Figure 1 shows the steady-state absorption and photoluminescence spectra $\left(\lambda_{\text {exc }}=470\right.$ $\mathrm{nm}$ ) of the stock solution of TIPS-Tc in an organic solvent (THF), freshly prepared TIPS-Tc nanoparticles dispersed in water and nanoparticles embedded in gelatin matrix. The major peaks in the absorption and emission spectra show the $0-0,0-1$ and 0-2 vibronic transitions of the singlet state in the molecule. The absorption spectrum remains almost unchanged when moving from dilute solutions to nanoparticles in solution or gelatin. The peak positions of the photoluminescence spectra of TIPS-Tc in molecular solutions and freshly prepared nanoparticles are comparable, along with the presence of broader spectral features of the latter in the lower energy region $(>600 \mathrm{~nm})$. However, in nanoparticles in gelatin matrix, we observe a prominent broad emission from a low- energy species which will be discussed later. The photoluminescence quantum efficiency (PLQE) measured for nanoparticles in gelatin matrix is $1.5 \%$ while dilute TIPS- Tc shows a high PLQE of $76 \%$. This is consistent with the presence of new non-radiative decay pathway for the singlet exciton in the nanoparticles. 
Figure $2 \mathrm{a}$ and $2 \mathrm{~b}$ show the transmission electron microscopy (TEM) images of freshly formed nanoparticles (measured on the day after synthesis) and aged nanoparticles, respectively. The former reveals that the initial size of the nanoparticles to be approximately $5 \mathrm{~nm}$ whereas the aged nanoparticles are approximately $30 \mathrm{~nm}$ in size. This size is comparable to the exciton diffusion length of triplet excitons in this system. ${ }^{11,40}$ The TEM images of the aged nanoparticles show interference fringes at their center revealing the crystalline nature of the core while the shell surrounding the core appears disordered. This means that these nanoparticles contain both crystalline and disordered regions, which have previously been studied separately in thin films and shown to have very different TT state decay dynamics ${ }^{24}$. The synthesis was repeated with high degree of reproducibility in terms of stability, morphology and size control of the nanoparticles. This allows us to probe a highly reproducible nanostructure, unlike thin films, where attempts to form both crystalline and disordered domains within the same films results in wide sample to sample variation. A more descriptive analysis of size and crystallinity of the nanoparticle based on TEM is included in the supporting information (figures 1 and 2). Crystal structure parameters such as d- spacing values are calculated directly through this analysis of interference fringes in TEM images and compared with the known crystal structure of the molecule.

\section{Identifying the correlated triplet- pair state}

A previously published study on singlet fission in solutions of TIPS- Tc showed the presence of an intermediate triplet pair (TT state), observed as a broad low energy band beyond $610 \mathrm{~nm}$ in its steady- state photoluminescence spectrum ${ }^{23}$. The TT state was identified in time-resolved photoluminescence and absorption measurements, with correlating kinetics. In a recent study, Stern et $a l^{24}$ report the influence of crystallinity on the decay of the TT state by a comparison of polycrystalline and disordered films of TIPS-Tc. It was found that TT states remained bound in polycrystalline films, but broke up to form free triplets on nanosecond timescales in disordered films ${ }^{24}$. The disordered films in the report had a weakly emissive TT state with a broad $620 \mathrm{~nm}$ centered emission spectrum, similar to that of the aged nanoparticles in the present study. This work will serve as the basis for the identification of spectral signatures in the current study on nanoparticles. 
Time-correlated single photon counting (TCSPC) measurements were performed and figure 3a shows the two-dimensional time-resolved emission scan (TRES) data of nanoparticles from 500 to $750 \mathrm{~nm}$. The kinetics of emission in the range of $540 \mathrm{~nm}$ to $700 \mathrm{~nm}$ indicate the presence of more than one species when excited at $470 \mathrm{~nm}$. Figure $3 \mathrm{~b}$ shows the kinetics of emission at $545 \mathrm{~nm}, 580 \mathrm{~nm}, 620 \mathrm{~nm}$ and $660 \mathrm{~nm}$, normalized to highest peak count. Notice that while the decay of $545 \mathrm{~nm}$ and $580 \mathrm{~nm}$ emission show a significant fast-decaying species (faster than instrument response) as well as a later slow decaying species (ns- lived), the decay of the $620 \mathrm{~nm}$ and $660 \mathrm{~nm}$ emission indicate a slower decaying (ns- lived), lower energy species. Also, note the delayed rise of about $1 \mathrm{~ns}$ in the emission at 620 and $660 \mathrm{~nm}$, which we will return to later.

The photoluminescence spectra obtained at different times (1- $20 \mathrm{~ns}$ ) normalized by area are shown in figure $3 \mathrm{c}$ which shows the presence of an isoemissive point. The presence of an isoemissive point, in area- normalized photoluminescence spectra suggests the presence of two distinct species. The initial-time spectra resemble that of singlet emission in dilute solutions, whereas spectra after $5 \mathrm{~ns}$ show a distinct redshifted, broad emission centred around $620 \mathrm{~nm}$ (figure 3c). SVD and global analysis by means of a genetic algorithm (GA) of the TRES data confirm the presence of two different species: a fast decaying singlet-like species with structured emission and a species formed at later times with a red-shifted broad emission (see SI figure 4a). This low energy species looks akin to the triplet- pair state (TT) reported in TIPS- Tc aggregates in solutions ${ }^{23}$. Comparing the emission spectrum of the solution and that of the nanoparticles in the present study (filled spectrum in figure $3 \mathrm{c}$ ), the broadening of the spectrum at lower energies in the latter suggests the formation of a comparable TT state. The time- resolved photoluminescence spectrum at $1 \mathrm{~ns}$ is thus assigned to singlet emission, similar to the molecular $S_{1}$ state in solutions of TIPS- Tc. At 5 ns and beyond the broad emission centered around $610 \mathrm{~nm}$ is assigned to TT states, like that observed in disordered films. The unusually long appearance of the singlet may be attributed to triplet-triplet annihilation and the re- formation of singlet or the presence of an equilibrium between singlets and TT states. 
The femtosecond transient absorption spectrum of the nanoparticles when pumped with a broadband pulse centred at $530 \mathrm{~nm}(<12 \mathrm{fs}$ pulse duration, 505-555 nm), is shown in figure $4 \mathrm{a}$. Spectra are shown for time delays from 40 fs to $180 \mathrm{ps}$, showing the typical spectral features expected from TIPS- Tc, as reported elsewhere ${ }^{23,24}$. This includes ground state bleach (GSB) at $545 \mathrm{~nm}$ (assigned from the corresponding absorption peak), stimulated emission (SE) at $580 \mathrm{~nm}$ (assigned from the photoluminescence) and photo- induced absorption (PIA) at higher wavelengths (assigned to multiple species). The existence of an isosbestic point in the transient absorption (TA) data is of particular interest. The negative nature of the isosbestic point here suggests the presence of a second species with absorption (PIA) in this region. Thus, kinetics of singlet states $\left(\mathrm{S}_{1}\right)$ obtained from the decay of $\Delta \mathrm{T} / \mathrm{T}$ at $580 \mathrm{~nm}$ (SE) also contains a small contribution from this second species. SVD sheds light on the presence of two distinct species and these were fit using GA, shown in figure $4 \mathrm{~b}$ along with spectra at $50 \mathrm{fs}$ (singlet-dominating) and $147 \mathrm{ps}$ (final time delay). The short-lived component is identified as the singlet whereas the longer lived component is spectrally similar to the TT state observed in reports of disordered films of TIPS$\mathrm{Tc}^{24}$, with a distinctive PIA peak around $620 \mathrm{~nm}$. The singlet has peaks at $580 \mathrm{~nm}$, $650 \mathrm{~nm}$ and a near-IR peak at $835 \mathrm{~nm}$ while the $620 \mathrm{~nm}$ peak and a broadened $835 \mathrm{~nm}$ peak are assigned to the TT state. Kinetics associated with spectral components from the GA are shown in the supplementary information (figure 8) which shows the rapid decay of the singlet matched by a fast rise of the TT state, within 200 fs. However the singlet also has a longer component has a lifetime of 17 ps from GA kinetics.SE decay kinetics at $580 \mathrm{~nm}$ (figure 4a, dark purple region) placing the singlet lifetime at $18 \mathrm{ps}$. Thus, global analysis is consistent with the raw data.

\section{Excitation energy dependence of early-time dynamics}

Transient absorption data, ranging from 1 ps to $1.2 \mathrm{~ns}$, of the nanoparticles when excited a $100 \mathrm{fs}$ narrowband pulse at $532 \mathrm{~nm}, 500 \mathrm{~nm}$ and $480 \mathrm{~nm}$ are shown in figure $5 \mathrm{a}$. Kinetics of the decay of $580 \mathrm{~nm}$ band $\left(\mathrm{SE}\right.$ of $\left.\mathrm{S}_{1}\right)$ gives a singlet lifetime of $12 \mathrm{ps}$ when excited at $532 \mathrm{~nm}, 17 \mathrm{ps}$ when excited at $500 \mathrm{~nm}$ and $28 \mathrm{ps}$ at $480 \mathrm{~nm}$ excitation. This is different from the singlet lifetime obtained from broadband excitation, $18 \mathrm{ps,} \mathrm{as} \mathrm{this} \mathrm{femtosecond} \mathrm{broadband} \mathrm{pump} \mathrm{(peak:} 530 \mathrm{~nm}$, FWHM: 50 
$\mathrm{nm}$ ) excites a broad ensemble of states, whereas the narrowband excitation excites specific sub-ensembles of electronic states. Importantly, when excited at $500 \mathrm{~nm}$ and $480 \mathrm{~nm}$, we see the emergence (by a delay of $1 \mathrm{~ns}$ ) of a spectrally different second species with increasing positive $\Delta \mathrm{T} / \mathrm{T}$ around $650 \mathrm{~nm}$ (purple region). We return to this feature below. Other changes on exciting with a higher energy excitation include: the near-IR peak at $835 \mathrm{~nm}$ becomes sharper and more structured (blue region), the presence of a sharper $900 \mathrm{~nm}$ peak (orange region) and $964 \mathrm{~nm}$ peak (grey region) of which the latter two were absent in $532 \mathrm{~nm}$ excitation. The $964 \mathrm{~nm}(1.29 \mathrm{eV})$ peak has been previously linked with the formation of a TT species in a previous report on TIPS-Tc ${ }^{24}$. However, in this study, we see the $964 \mathrm{~nm}$ peak at times as early as $1 \mathrm{ps}$ which is indicative of prompt formation of the TT state. Global analysis of the data by GA identifies two distinct species when excited at each of these three energies, as shown in figure $6 \mathrm{a}$ and $6 \mathrm{~b}$. Based on previous reports ${ }^{23,24}$ and the fs- TA data presented in figure 4 we assign these two species to the singlet and correlated TT states.

Figure $5 \mathrm{~b}$ shows the kinetics associated with identified singlets and TT states at these three excitation energies. The singlet lifetime is found to increase from 9 ps to 22 ps when excited at $532 \mathrm{~nm}$ and $480 \mathrm{~nm}$, respectively. The delayed rise- time of the TT population, deconvoluted via the GA, are 2 ps when excited at $532 \mathrm{~nm}, 4$ ps when excited at $500 \mathrm{~nm}$ and $7 \mathrm{ps}$ at $480 \mathrm{~nm}$ excitation. The rise of the prompt component of the TT state (under $200 \mathrm{fs}$ ), see in figure 4, is not observable in these experiments due to the limited time resolution. The $\mathrm{S}_{1}$ and TT kinetics obtained from GA of the ps- TA data shown in figure 5 are normalized relative to $\mathrm{S} 1$ to give the relative TT yield. In other words, the normalized units for the TT population will thus give insights into yield of TT with respect to singlets. We see that TT yield is the highest $(<0.8)$ when excited at $532 \mathrm{~nm}$ and the lowest $(<0.4)$ when excited at $480 \mathrm{~nm}$. Comparison of the PIA intensities of TT (600 nm band) with respect to the singlet $(650 \mathrm{~nm}$ band) in these experiments shows the same trend as explained in the supporting information, table 1 .

We note the disparity between the delayed TT rise- time and singlet lifetime. This could have multiple causes; (1) Excited singlet states go down two different 
pathways, a fraction undergoing TT formation while the rest relaxing into ground state, which would be supported by the varying TT yield observed for different excitation wavelengths (2) The nature of excited states (mixture of singlet and TT) governs the equilibrium between singlet and TT states, thus causing seemingly noncorrelated TT rise- time and singlet decay constant. This would explain why the respective time constants vary with excitation energy as well (3) TT states formed undergo annihilation and re- form singlet states giving rise to longer singlet lifetimes that do not correlate with TT rise- times. However, within the current data set it is difficult to establish which of these possibilities is dominant. We note that the difference in these excitation energies is only $150-200 \mathrm{meV}$ and thus it is surprising to observe such pronounced differences in the resulting dynamics.

While the singlet spectral features at the different pump wavelengths are the same as in the femtosecond data shown in figure 4 , the TT spectrum is found to change with excitation energy. For $480 \mathrm{~nm}$ excitation, a delayed positive feature appears near 650 $\mathrm{nm}$ (1 ns spectrum, see figure 5a purple region), which is less pronounced when excited at $500 \mathrm{~nm}$ and completely absent under $532 \mathrm{~nm}$ excitation. The delayed rise of this feature matches the delayed rise of emission from the low energy species $(>620$ $\mathrm{nm}$ ) observed in the TRES data (figure $3 \mathrm{~b}$ ). This delayed broad emission from the TRES data at longer times has been identified as emission from the TT state (as discussed before). Consequently, along with supportive evidence from GA analysis, the late-forming species in the TA experiment is assigned the TT state. This means that the positive band around $630 \mathrm{~nm}$ in the TA spectra, when excited at higher energies, arises due to stimulated emission from the TT state, suggesting that the TT state can now directly relax to the $\mathrm{S}_{0}$ state, as illustrated in figure $6 \mathrm{c}$. As the TT state cannot be directly photo- excited from the ground state geometry of $\mathrm{S}_{0}$, this implies that the molecules are no longer in the ground state geometry. This is not surprising as the excited state wavepacket will move (on the potential energy surface) away from the Frank-Condon point following the initial photoexcitation. The change in molecular geometry can enable previously forbidden transitions to become allowed.

Emission from the TT state has been observed in acenes ${ }^{23,24,41,42}$. Combined with twophoton photoemission studies ${ }^{43,44}$ this suggests that the initially prepared singlet states 
have some multiexciton character. It therefore follows that the TT state has finite $\mathrm{S}_{1}$ character. As such, a Herzberg-Teller (HT) type mechanism has recently been invoked to explain direct PL from TT states in acenes ${ }^{45}$. Considering a four orbital basis of the pair excitation states ${ }^{46}$, this necessitates an inter-chromophore exchange coupling which breaks the degeneracy between singlet and quintet TT pairs ${ }^{47}$. Recent studies on films of TIPS- tetracene place an upper bound of $4.2 \mathrm{meV}$ on this exchange energy ${ }^{22}$. We note that the multiexciton states in the nanoparticles seen as a function of pump energy are unlikely to be of quintet character because (1) they are formed in picoseconds as opposed to slower (nanoseconds) formation of quintets previously observed in TIPS-tetracene films ${ }^{22}$ and (2) they decouple into free triplets in nanoseconds as discussed later in this work.

Here we observe not just PL, but pump energy dependent stimulated emission directly from the TT states. When the system is excited with different energies, the initial Frank-Condon point is varied, see figure $6 \mathrm{c}$, and hence the relaxation pathway for the excited state changes. Our results show that depending on where on the excited state potential energy surface the initial wavepacket is formed, the evolution of $\mathrm{S}_{1}$ to TT can take longer, as evidenced by the change in decay rate of the stimulated emission from $\mathrm{S}_{1}$, and that the TT state that the system relaxes to can be completely different with different yields.

Further to excitation energy dependent ps- TA experiments above, time-resolved photoluminescence measurements were done at $530 \mathrm{~nm}, 500 \mathrm{~nm}$ and $480 \mathrm{~nm}$ excitations, leading to the formation of dark and/or bright TT species selectively at different energies (supplementary information, figure 5). While previously assigned broad emission from the TT state around $620 \mathrm{~nm}$ is present when excited at $480 \mathrm{~nm}$ and $500 \mathrm{~nm}$, at $532 \mathrm{~nm}$ the photoluminescence spectrum is dominated by emission from the singlet which also differ in kinetics from the former. The kinetics of singletlike and TT- like regions from these measurements confirm the presence of multiple species and more importantly, different TT states being formed when excited at different energies. 
In order to gain a more complete picture of the effect morphology and the generality of the above presented picture for multiple TT state formation, we have conducted picosecond narrowband transient absorption experiments at different excitation energies, on disordered thin films of TIPS-T (see SI figure 7). For excitation at $532 \mathrm{~nm}$, the TT formed has a flat absorption beyond $600 \mathrm{~nm}$. However, at $480 \mathrm{~nm}$ excitation the TT state spectrum shows a positive- feature around $650 \mathrm{~nm}$ which is akin to the spectra feature assigned to stimulated emission from the TT state in the case of the nanoparticles. We note that these features are more difficult to detect in the thin films, due to signal to noise considerations, which makes the nanoparticle system better suited to examine these processes. However, the observation of different TT states forming in thin films samples as well, points to the generality of this important phenomena, one that has not been considered in studies of singlet fission till date. A similar excitation energy dependence thus observed in the formation of different TT states shed light on generalizing the formation of multiple TT formation pathways that could sometimes even lead to bright TT states, across morphologies. This a very compelling piece of evidence that suggests generalizing multiple TT formation pathways that are accessed vibrationally via change in excitation energy, in different morphologies of this endothermic singlet fission system.

\section{TT to free triplets}

Transient absorption spectra at longer delay times (1 ns to $5 \mu \mathrm{s}$ ) when the nanoparticles were excited at $532 \mathrm{~nm}$ are shown in figure 7a. SVD/DAS showed the presence of two distinct components, shown in figure $7 \mathrm{~b}$, identified as TT state and free triplets $\left(\mathrm{T}_{1}\right)$ by comparing the current data to previously published data ${ }^{24}$ on disordered and polycrystalline films of TIPS- Tc and solution triplet sensitization measurements, as discussed later. The TT state has distinct negative peaks at $620 \mathrm{~nm}$ (purple region), $835 \mathrm{~nm}$ (blue region), $968 \mathrm{~nm}$ (grey region) and $1000 \mathrm{~nm}$ and is converted to free triplets with major negative peaks at $835 \mathrm{~nm}, 890 \mathrm{~nm}, 925 \mathrm{~nm}, 972$ $\mathrm{nm}$ and $1000 \mathrm{~nm}$. The TT spectrum at $1 \mathrm{~ns}$ from this experiment matches the spectrum at $1 \mathrm{~ns}$ and beyond in the picosecond experiment, and have sharper bands compared to that of the singlet in the near- IR region. The rise of new bands at 890 $\mathrm{nm}$ and $925 \mathrm{~nm}$ (orange region) and spectral shift from $964 \mathrm{~nm}$ to $972 \mathrm{~nm}$ (grey 
region) mark the dissociation of TT states and formation of free triplets by $100 \mathrm{~ns}$. These free triplets are present for longer than $100 \mu \mathrm{s}$, despite the confined nature of the nanoparticle. This long triplet lifetime is beneficial for device applications of singlet fission.

As previously described, from time-resolved photoluminescence measurements, the broad peak above $610 \mathrm{~nm}$ is assigned to the TT state, while the $545 \mathrm{~nm}$ peak is assigned to the singlet. Figure 8 shows the kinetics of emission at $545 \mathrm{~nm}$ and $660 \mathrm{~nm}$ overlapped with the decay of the 965-970 nm TA band assigned to TT at early times and free triplets at later times. The kinetics of the $660 \mathrm{~nm}$ emission from the TT state matches the TA kinetics until about $10 \mathrm{~ns}$ after which they diverge due to decoupling to form free triplets. This is consistent with the timescale for the formation of free triplets, and further confirms the spectral assignments. Table 1 summarises all spectral assignments and time constants associated with different species, along with the experimental conditions for them.

Previously, it has been shown that TT states form rapidly in both polycrystalline and disordered films, but that in polycrystalline films TT states remained bound out to $\mu \mathrm{s}$ timescales. In contrast, in disordered films TT states decoupled to form free triplets on ns timescales ${ }^{24}$. The nanoparticles studied here have a crystalline core and disordered shell, as shown in figure $2 \mathrm{~b}$. As singlet excitons will form in both the disordered and crystalline parts of the nanoparticle upon photoexcitation, it is expected that TT states will form in both phases on ps and sub-ps timescales. But we do not observe any features consistent with long-lived $(\mu \mathrm{s})$ TT states, see figure 7 . This suggests the possibility that TT states formed within the crystalline core maybe diffusing into the disordered exterior regions, where they decouple to form free triplets. The diffusion of TT states is an unexplored area and our results hint that this process might be possible and a very important step in the generation of free triplets.

\section{Conclusions}

In this work, we have synthesized and studied the dynamics of nanoparticles of TIPSTc, an endothermic singlet fission system. The formation of nanoparticles with a 
crystalline core and disordered exterior region creates a unique platform to study the effects of morphology on the dynamics of TT states. Using steady-state and timeresolved PL and transient absorption spectroscopy we identify the formation of the TT state, which has distinct spectral signatures in the transient absorption and a broad PL beyond $610 \mathrm{~nm}$. These spectral signatures are consistent with previous assignments in thin films of TIPS-Tc ${ }^{24}$. The initial rise of the TT state occurs with a sub-500 fs rate constant. The subsequent rise of the TT states occurs with a rate of 2, 4 and $7 \mathrm{ps}$, whereas the decay of the $S_{1}$ occurs, as judged by the loss of stimulated emission, with a rate constant of 12,17 and 28ps, as the excitation wavelength is varied from $532 \mathrm{~nm}$, to $500 \mathrm{~nm}$ to $480 \mathrm{~nm}$. The final TT states formed at these different excitation energies show very different spectral signatures. These large differences imply that the initial relaxation away from the Frank-Condon point controls the choice of pathway for singlet fission and that multiple distinct intermediates within the TT manifold are present in the process. This has important implications for the mechanistic understanding of singlet fission which has previously considered the TT state as a single species. The ability to access multiple TT states, even in endothermic fission systems, on ultrafast fast timescales could allow singlet fission to outcompete radiative and non-radiative recombination in TIPS-Tc. These results also point to the importance role vibrational dynamics play in mediating the fission process and strong parallels can be drawn with the photophysics of molecular systems such as rhodopsin where the yield and reaction pathway are strongly dependent on the shape of the potential energy surface and the way the wavefunction approaches the state crossing, rather than simple energetics and couplings between states in the ground state geometry of the system. Future theoretical work will need to explicitly consider the possibility of multiple trajectories along different potential energy surfaces, in order to provide a more complete description of the fission process. On nanosecond timescales, we observe the evolution to the TT states to free triplets, suggesting that TT states may be diffusing from the crystalline core of the particle to the disordered exterior, where the decoupling of TT into free triplets is favored, as seen in disordered films before ${ }^{24}$. This highlights the importance of TT state diffusion to the fission process, something that has until now been unexplored. It is possible that this TT state diffusion may also play a role in the crystallite size dependence of fission seen in other systems ${ }^{27,32}$. In future, careful control of morphology, like that undertaken in 
organic photovoltaics, could result in optimized nanostructures where the directed flow and dissociation of TT states is achieved to maximize the generation of free triples for device applications.

\section{Acknowledgement}

The authors acknowledge the EPSRC and the Winton Programme for the Physics of Sustainability for funding. A.T. thanks the Dr. Manmohan Singh scholarship, St John's College, University of Cambridge for funding.

\section{Author Information Notes}

\section{Corresponding Author}

*ar525@cam.ac.uk

\section{ORCID}

0000-0003-4261-0766

\section{Associated Content}

Supporting information document (*.docx) contains description and data (figures) on the comparison of correlated triplet pair- forming and decoupling kinetics among different morphologies. Kinetics plot fits to show sub- 200fs rise-time of the TT state and free triplet formation, information regarding crystallinity of the nanoparticles, excitation energy dependent time-resolved photoluminescence data, evidence on excitation energy dependent TT formation in disordered films etc. are included as well. 


\section{Figures}

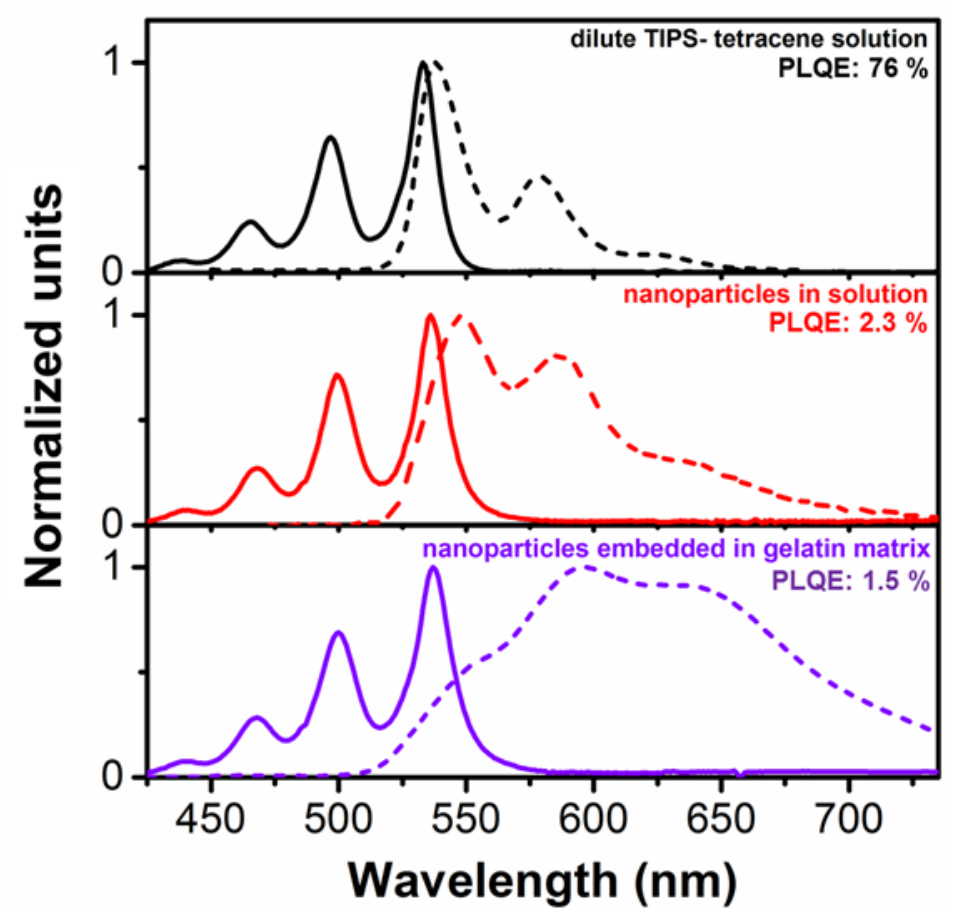

Figure 1. Normalized steady- state absorption (solid lines) and photoluminescence (dotted lines) spectra of diluted TIPS- Tc in tetrahydrofuran (black), TIPS- Tc nanoparticles suspended in water (red) and TIPS- Tc nanoparticles embedded in gelatin polymer matrix in solid state. For photoluminescence measurements, the samples were excited at $470 \mathrm{~nm}$. Photoluminescence quantum efficiency (PLQE) of dilute TIPS- Tc solution was measured to be $76 \%$, nanoparticles in solution $2.3 \%$ and nanoparticles in gelatin matrix was $1.5 \%$. 
(a)

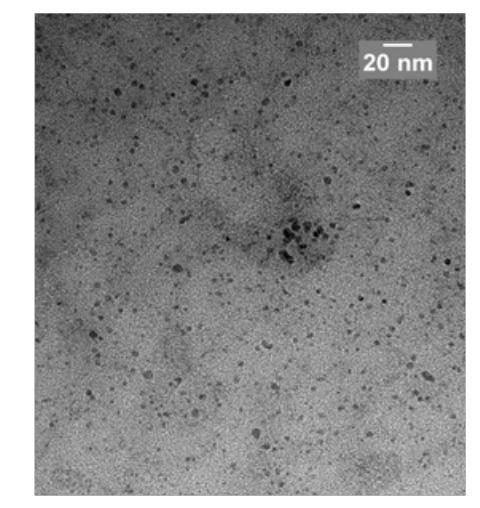

D-spacing values calculated from FFT of interference fringes in the highlighted core of the nanoparticle: d: $0.17,0.21,0.31 \mathrm{~nm}^{-1}$

(b)

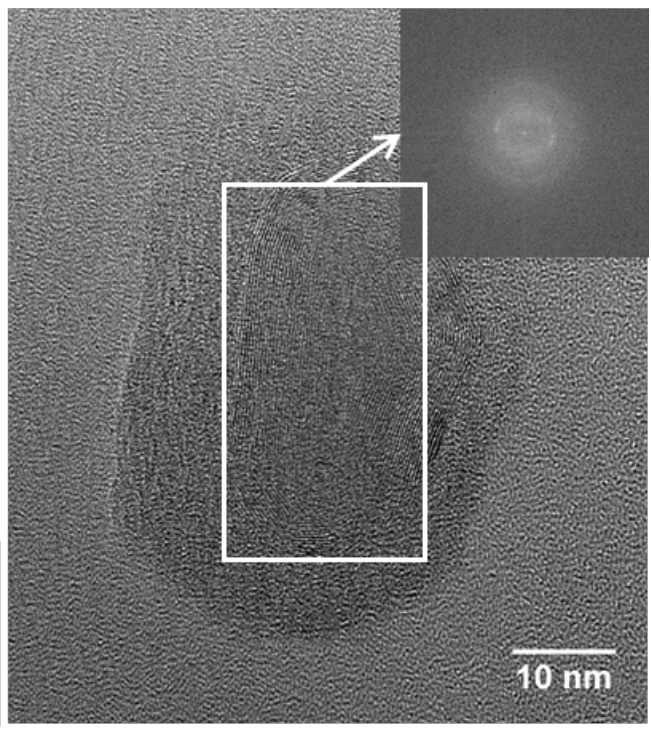

Figure 2. Transmission electron micrographs of TIPS- Tc nanoparticles: (a) one day post- synthesis, and (b) 75 days post- synthesis. The aged nanoparticle has a crystalline core and disordered exterior. All figures are calibrated to the scale bar shown in the index. Notice interference fringes at the core of the nanoparticle in (b) which shows that the core crystallises upon ageing while the exterior remains disordered. Analysis of these fringes by Fourier function transform (FFT) produces a diffraction image of the reciprocal lattice (inset) with d- spacing values, which are shown in the figure. See SI for further discussion. 
(a)
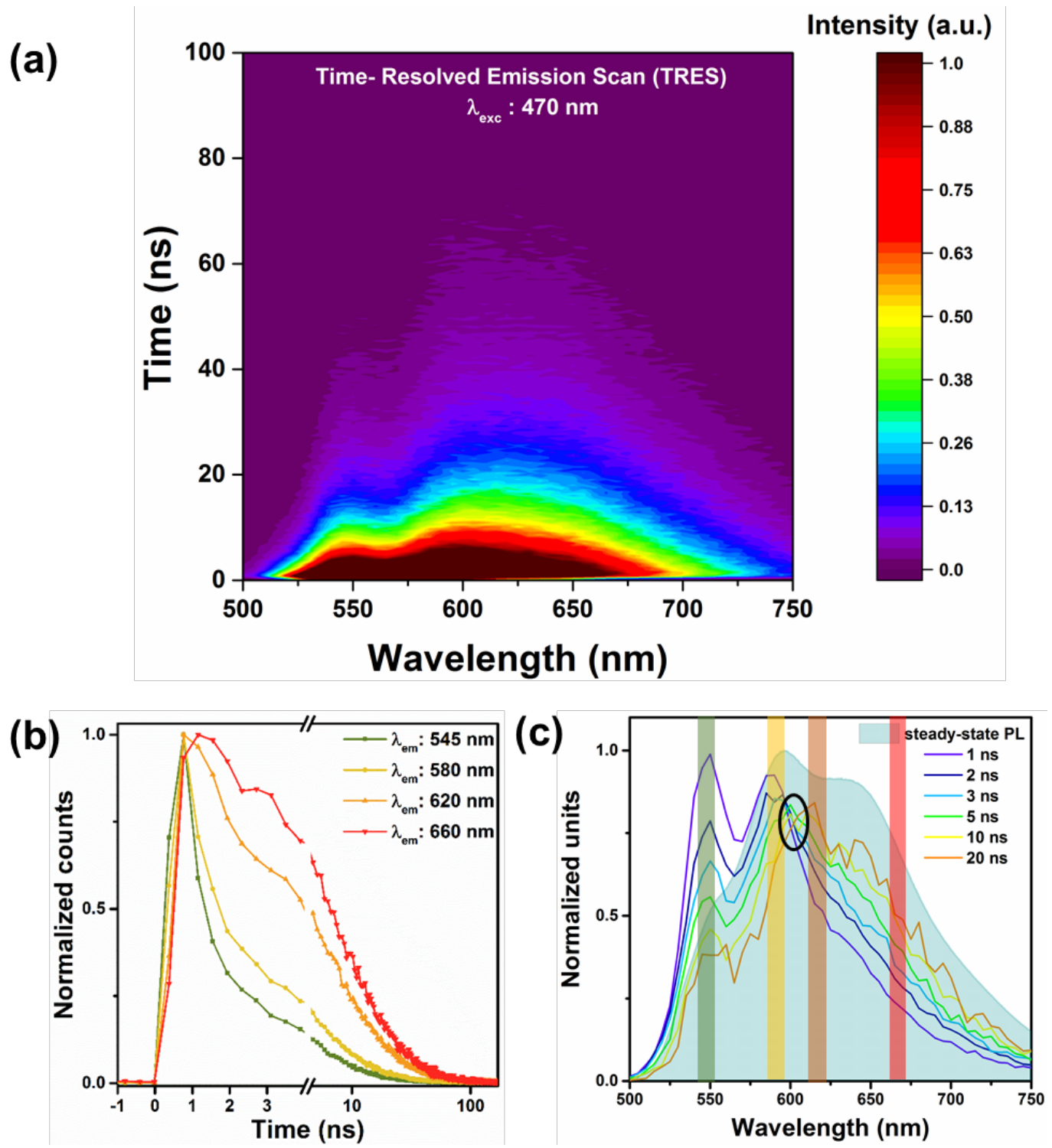

Figure 3. (a) Two- dimensional time- resolved emission scan (TRES) data of the nanoparticles embedded in gelatin matrix when excited at $470 \mathrm{~nm}$. Singlet emission peaks at $545 \mathrm{~nm}, 580 \mathrm{~nm}$ and TT-singlet 'mixed' emission beyond $600 \mathrm{~nm}$ as well as how long they live can be seen in this 2D map. (b) shows the kinetics of emission at $545 \mathrm{~nm}, 580 \mathrm{~nm}$ which are primarily singlet, $620 \mathrm{~nm}$ and $660 \mathrm{~nm}$ which are in the TT region. Notice the longer rise time in the emission of TT associated region. (c) shows the spectra of components at $1 \mathrm{~ns}$ to $20 \mathrm{~ns}$ in the time-resolved measurement and the steady- state spectrum (blue shaded) of the nanoparticles. A largely singlet like emission is predominant at $1 \mathrm{~ns}$ while at longer timescales most of the emission is red shifted indicating the presence of a different emissive species. All plots have been normalized by area. The red- shifted emitting, later forming component has been assigned to the TT state. 


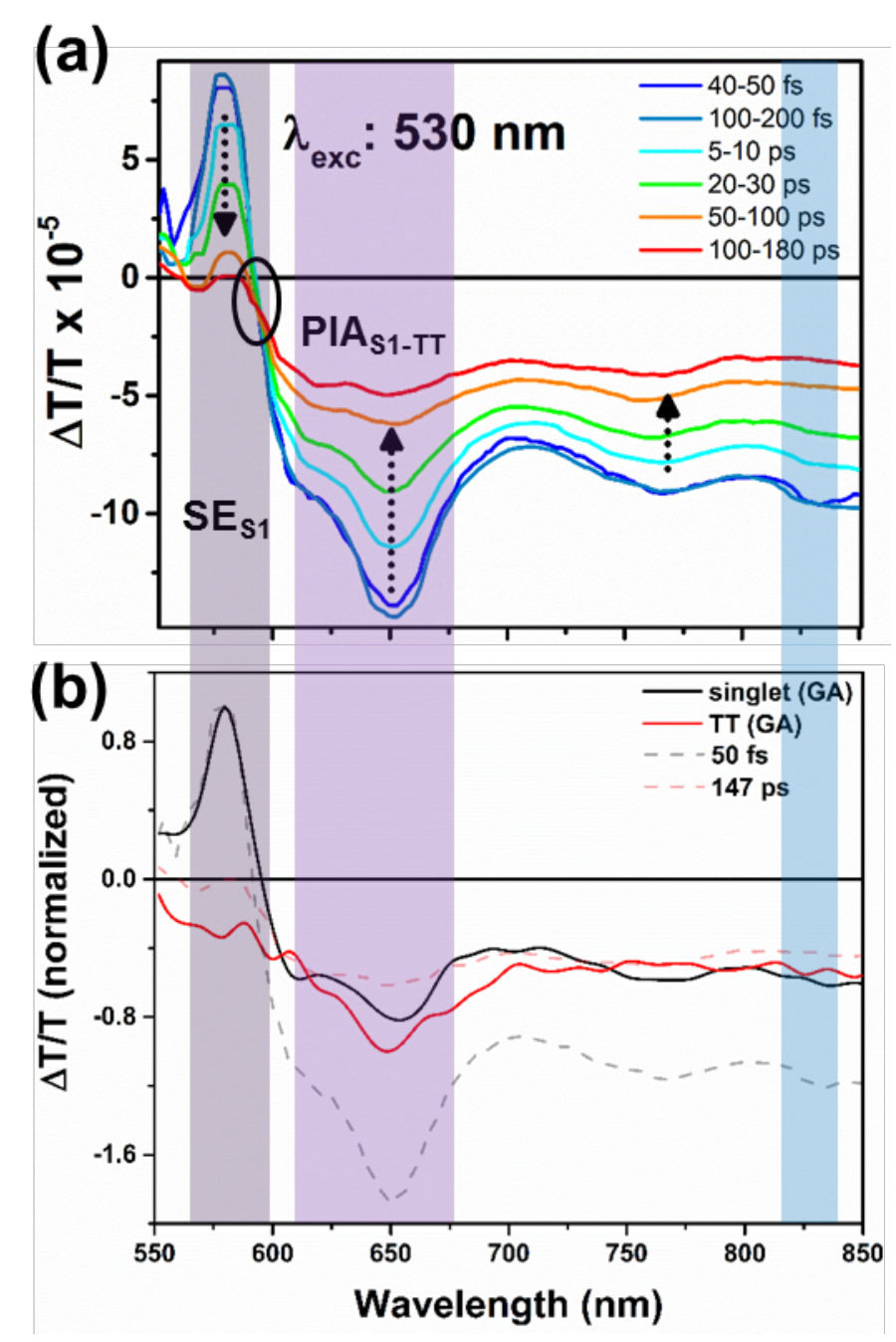

Figure 4. Transient absorption spectra when excited at $530 \mathrm{~nm}$ with time delays from 40 fs to 180 ps. (a) Stimulated emission and photo- induced absorption of the singlet decay with a lifetime of 12 ps. The arrows represent the said decays of singlet bands. Shaded bands represent these processes as marked in figure. The presence of an isobestic point (marked by black circle) indicates two species and DAS confirms that. Figure (b) shows the spectral components from GA associated with singlet (blue) and TT state (black). The dotted traces in figure $4 \mathrm{~b}$ shows spectra at 50 fs (singletdominated) and 147 ps (TT dominated) for comparison. 

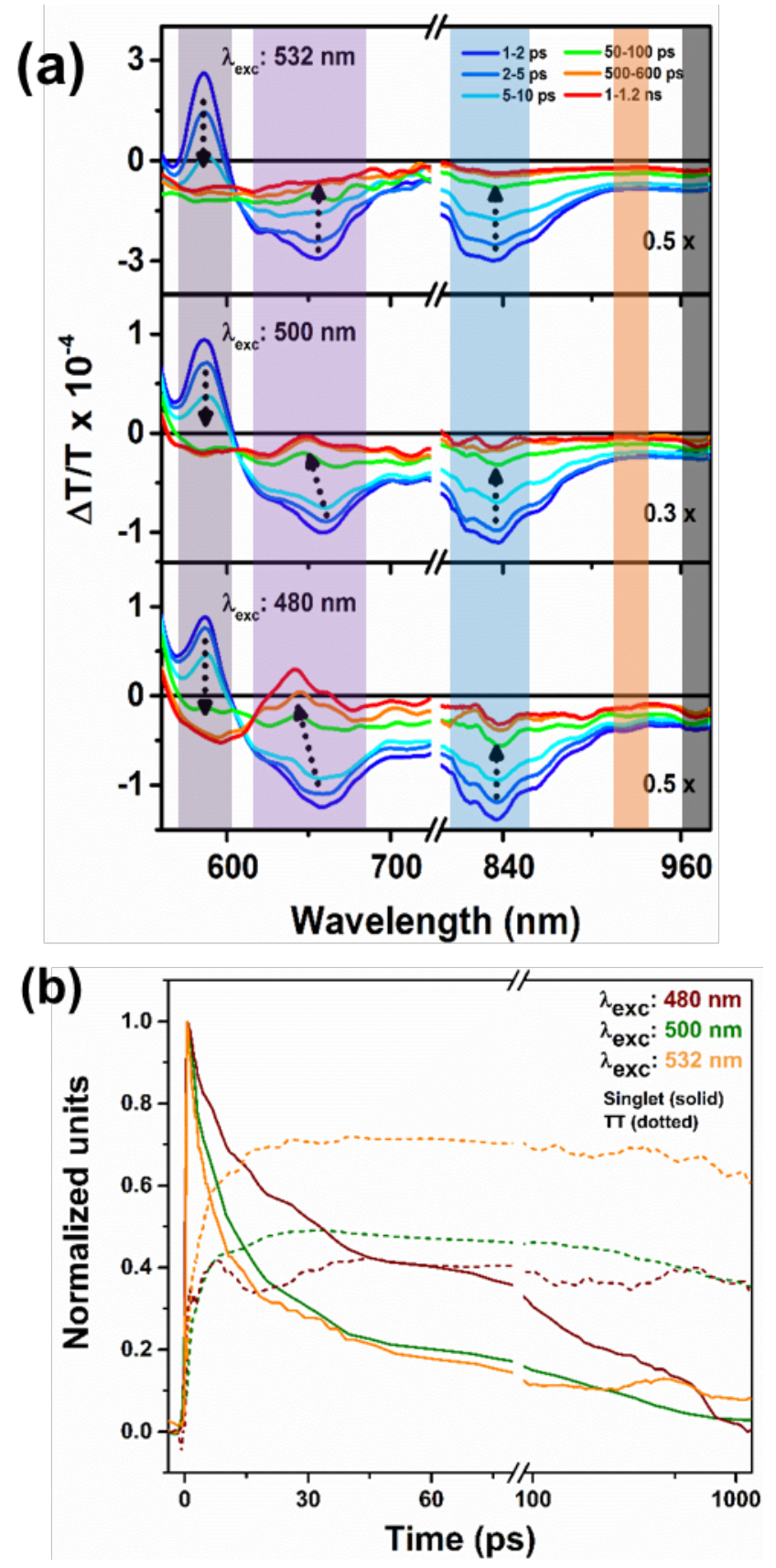

Figure 5. Transient absorption spectra (a) when excited at pump energies $532 \mathrm{~nm}$, $500 \mathrm{~nm}$ and $480 \mathrm{~nm}$ with time delays 1 ps to $1.2 \mathrm{~ns}$. (b) shows the kinetics of singlet and TT states obtained from global analysis of data shown in (a) using a genetic algorithm (GA) at excitation wavelengths $532 \mathrm{~nm}$ (orange), $500 \mathrm{~nm}$ (green) and 480 $\mathrm{nm}$ (red). As pump energies increase from $532 \mathrm{~nm}$ to $480 \mathrm{~nm}$, the rate of singlet decay and TT formation decreases, more prominently at early times. Kinetics have been normalized with respect to the singlet maximum giving insight into the TT yield which is the highest at $532 \mathrm{~nm}$ excitation and the lowest at $480 \mathrm{~nm}$ excitation. 
(a)

(b)
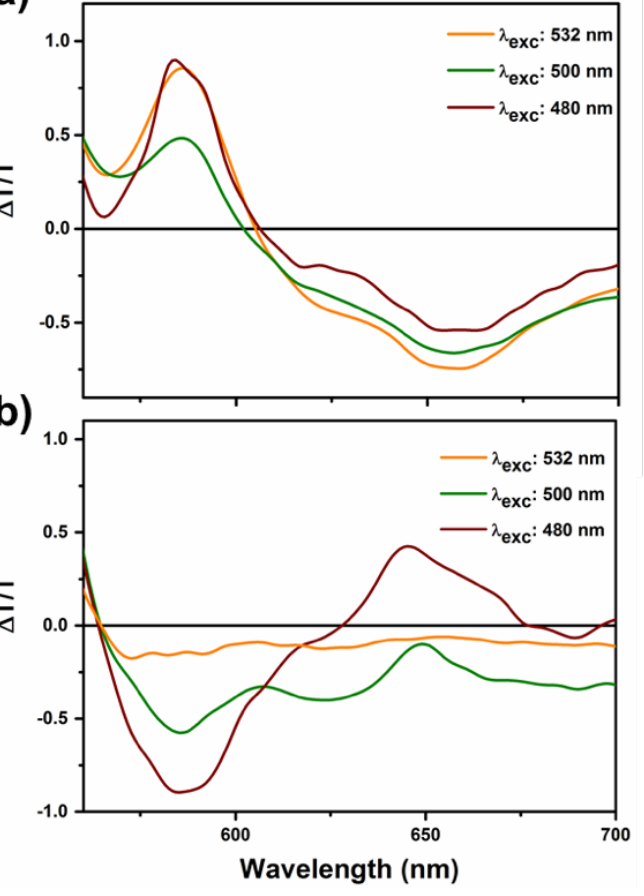

(c)

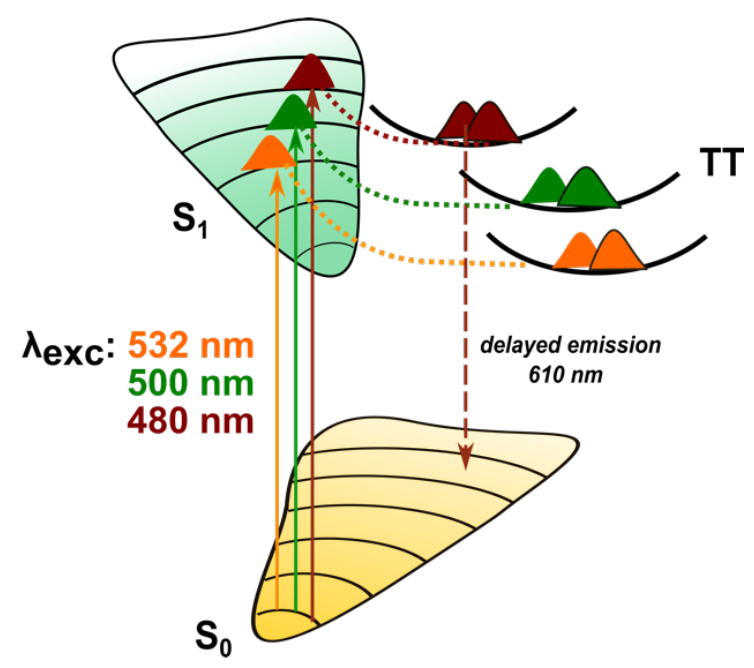

Figure 6. Spectral deconvolution of the ps TA data shown in figure 5a at excitation energies $532 \mathrm{~nm}$ (orange), $500 \mathrm{~nm}$ (green) and $480 \mathrm{~nm}$ (red) done by means of a genetic algorithm (GA) predicted two distinct species shown in (a) and (b). The first GA predicted spectra shown in (a) greatly resemble the S1 spectrum and matches the experimental singlet lifetime of about 12 ps. The singlet spectral shape remains unaltered while the SE and PIA peaks at $580 \mathrm{~nm}$ and $650 \mathrm{~nm}$ vary in intensity ratio across the three. The second GA predicted spectrum at $480 \mathrm{~nm}$ resembles the $1 \mathrm{~ns}$ timeslice in the data shown in figure $5 \mathrm{a}$, with a positive feature at $610 \mathrm{~nm}$. From timeresolved photoluminescence data, we assigned the emission at $610 \mathrm{~nm}$ as a lowenergy TT species with a rise time of $1 \mathrm{~ns}$. The second GA species shown in (b) is thus assigned to the TT state. (c) shows a cartoon of how TT generation varies with pump- energy. At the highest pump- energy $(480 \mathrm{~nm})$, the TT state formed shows a delayed emission at $610 \mathrm{~nm}$ while TT states formed at lower pump- energies are nonemissive and dissociates to form triplets. The TT state energies are not drawn to scale. 

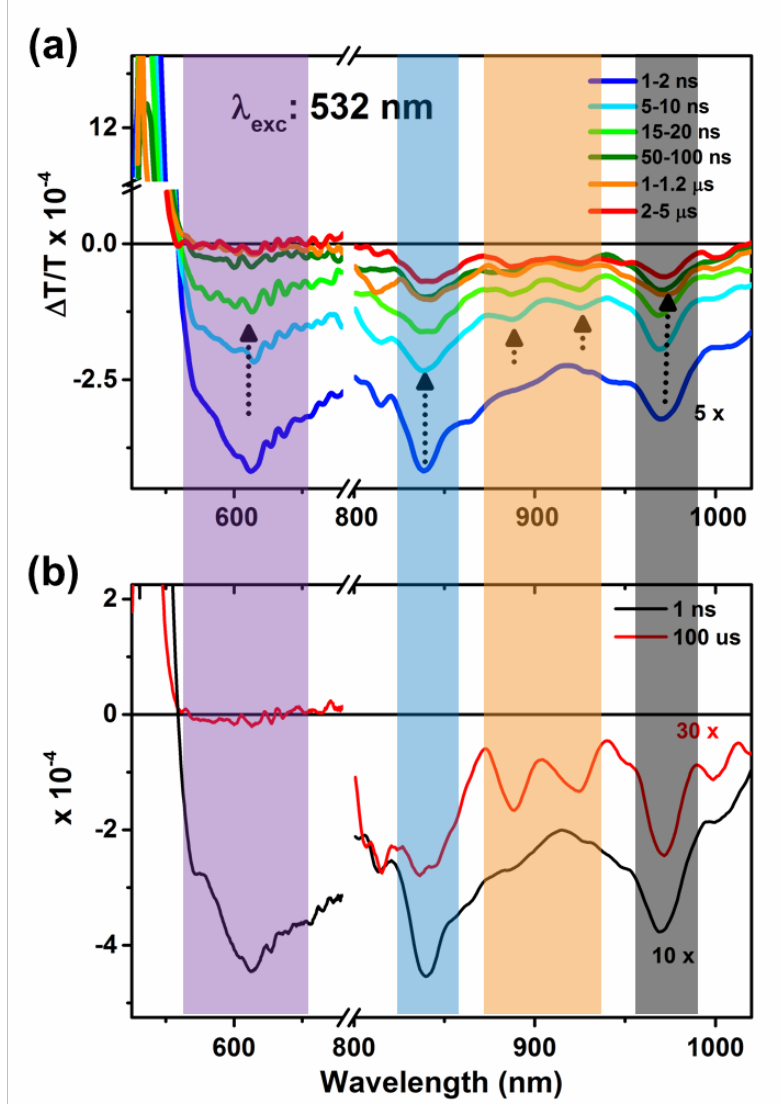

Figure 7. Transient absorption spectra (a) when excited at $532 \mathrm{~nm}$ with time delays 1 ns to $5 \mu \mathrm{s}$. (b) DAS of TT state (black) and free triplets (red) obtained from singlevalued decomposition (SVD) analysis of the TA data. Triplet peaks at $889 \mathrm{~nm}$ and $925 \mathrm{~nm}$ appear as early as under $10 \mathrm{~ns}$ and live beyond instrument limit $(100 \mu \mathrm{s})$. 


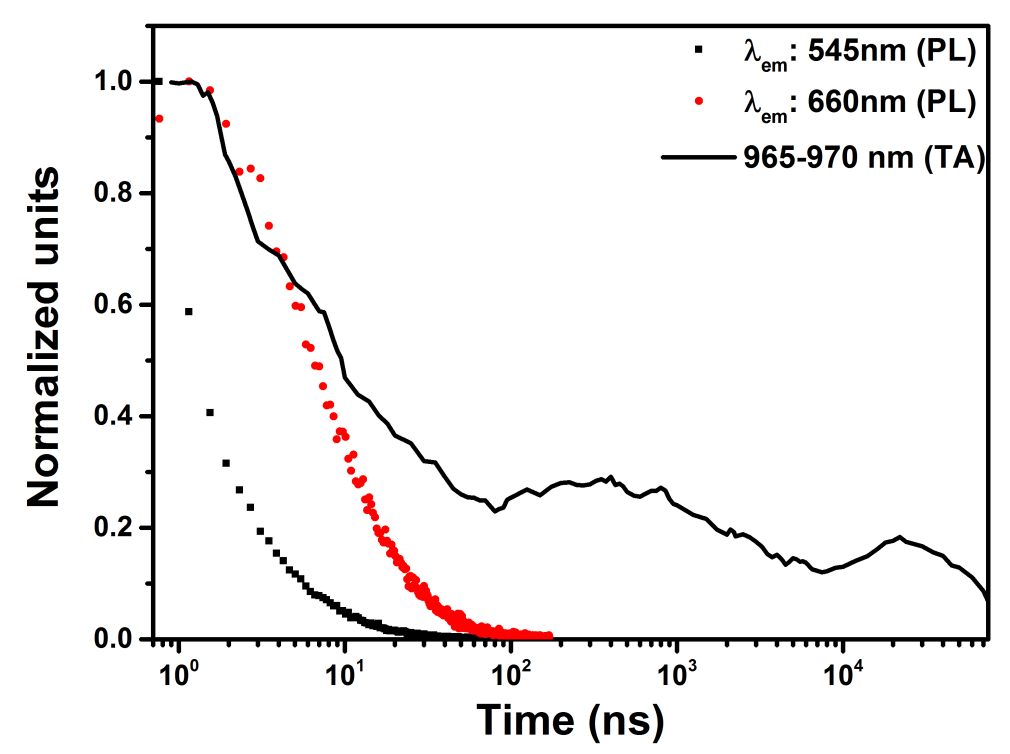

Figure 8. Kinetics from photoluminescence emission at $545 \mathrm{~nm}$ (singlet) and $660 \mathrm{~nm}$ (TT state) and the kinetics from ps and ns transient absorption measurements in the TT- free triplet region $965-970 \mathrm{~nm}$. The shift in the position of the $965 \mathrm{~nm}$ peak from ps to us is superimposed on to the kinetics mentioned above. The photoluminescence component at $620 \mathrm{~nm}$ and above is attributed to the TT state and kinetics of emission at $620 \mathrm{~nm}, 660 \mathrm{~nm}$ overlap greatly with kinetics obtained from TA of the $965 \mathrm{~nm}$ band which represents conversion of TT to free triplets up to sub- 10 ns. Marker bands of free triplets rise from 3 ns TA spectrum as discussed previously and this causes the deviation in PL and TA kinetics beyond sub- $10 \mathrm{~ns}$ timescale. The kinetics of $545 \mathrm{~nm}$ emission, predominantly from the singlet is faster as shown in the figure and it clearly does not have any TT component. For time-resolved emission scan, the sample was excited at $470 \mathrm{~nm}$ and for TA measurements, the sample was pumped at $532 \mathrm{~nm}$ and probed in the near- IR region. 


\begin{tabular}{|c|c|c|c|c|c|}
\hline & $\begin{array}{c}f_{S} T A \\
\lambda_{\text {exc }} \text { : } \\
\text { Broadband } \\
\text { (FWHM of } \\
50 \mathrm{~nm}) \\
\text { centred at } \\
530 \mathrm{~nm}\end{array}$ & $\begin{array}{c}p s T A \\
\lambda_{\text {exc }}: 532 \\
n m\end{array}$ & $\begin{array}{c}p s T A \\
\lambda_{\text {exc }} \cdot 500 \\
n m\end{array}$ & $\begin{array}{c}p s T A \\
\lambda_{\text {exc }}: 480 \\
n m\end{array}$ & $\begin{array}{c}n s T A \\
\lambda_{\text {exc }}: 532 \\
n m\end{array}$ \\
\hline $\begin{array}{c}S_{1} \text { to } T T \\
\text { decay }\end{array}$ & $18 \mathrm{ps}$ & $12 \mathrm{ps}$ & $17 \mathrm{ps}$ & $28 \mathrm{ps}$ & \\
\hline $\begin{array}{l}\text { TT rise-time } \\
\text { (beyond } \\
\text { sub- } 500 f_{S} \\
\text { rise) }\end{array}$ & & $2 \mathrm{ps}$ & $4 \mathrm{ps}$ & $7 \mathrm{ps}$ & \\
\hline $\begin{array}{c}\text { TT to } T_{1} \\
\text { decay }\end{array}$ & & & & & $3 \mathrm{~ns}$ \\
\hline$T_{1}$ lifetime & & & & & $>2 \mu \mathrm{s}$ \\
\hline
\end{tabular}

Table 1. List of experimental data used is tabulated along columns and processes whose time constants are estimated along rows. All time constants were calculated from raw kinetics data (marked in bold under Spectral features) which match closely with global population kinetics fit by means of genetic algorithm (GA) and decay associated spectral analysis (DAS). Singlet to TT conversion is slower at higher pump energy, while the TT rise time is not affected considerably. TT converts into free triplets with a lifetime of $3 \mathrm{~ns}$ and triplets ling longer than $2 \mu \mathrm{s}$. 


\section{References}

(1) Shockley, W.; Queisser, H. J. J. Appl. Phys. 1961, 32, 510.

(2) Swenberg, C. E.; Geacintov, N. E. Exciton Interactions in Organic Solids; 1973; Vol. 18.

(3) Pope, M.; Swenberg, C. E. Electronic Processes in Organic Crystals and Polymers; 1999.

(4) Hanna, M. C. \& Nozik, A. J. J. Appl. Phys. 2006, 100, 74510.

(5) Jadhav, P. J.; Brown, P. R.; Thompson, N.; Wunsch, B.; Mohanty, A.; Yost, S. R.; Hontz, E.; Van Voorhis, T.; Bawendi, M. G.; Buloviä, V.; Baldo, M. A. Adv. Mater. 2012, 24, 6169.

(6) Ehrler, B.; Wilson, M. W. B.; Rao, A.; Friend, R. H.; Greenham, N. C. Nano Lett. 2012, 12, 1053.

(7) Smith, M. B.; Michl, J. Annu. Rev. Phys. Chem. 2013, 64, 361.

(8) Tayebjee, M. J. Y.; Gray-Weale, A. A.; Schmidt, T. W. J. Phys. Chem. Lett. 2012, 3, 2749.

(9) Wilson, M. W. B.; Rao, A.; Ehrler, B.; Friend, R. H. Acc. Chem. Res. 2013, 46, 1330 .

(10) Wilson, M. W. B.; Rao, A.; Johnson, K.; Ge, S.; Pietro, R.; Clark, J.; Friend, R. H. J. Am. Chem. Soc. 2013, 135, 16680.

(11) Wan, Y.; Guo, Z.; Zhu, T.; Yan, S.; Johnson, J.; Huang, L. Nat. Chem. 2015, 7, 785 .

(12) Roberts, S. T.; McAnally, R. E.; Mastron, J. N.; Webber, D. H.; Whited, M. T.; Brutchey, R. L.; Thompson, M. E.; Bradforth, S. E. J. Am. Chem. Soc. 2012, 134, 6388 .

(13) Akdag, A.; Havlas, Z.; Michl, J. J. Am. Chem. Soc. 2012, 134, 14624.

(14) Wen, J.; Havlas, Z.; Michl, J. J. Am. Chem. Soc. 2015, 137 (1), 165.

(15) Musser, A. J.; Maiuri, M.; Brida, D.; Cerullo, G.; Friend, R. H.; Clark, J. J. Am. Chem. Soc. 2015, 137, 5130.

(16) Wang, C.; Schlamadinger, D. E.; Desai, V.; Tauber, M. J. ChemPhysChem 2011, 12, 2891.

(17) Klenina, I.; Makhneva, Z.; Moskalenko, A.; Gudkov, N. D.; Bolshakov, M.; Pavlova, E.; Proskuryakov. Biochemistry 2014, 79, 235.

(18) Burdett, J. J.; Bardeen, C. J. Acc. Chem. Res. 2013, 46, 1312.

(19) Burdett, J. J.; Muller, A. M.; Gosztola, D.; Bardeen, C. J. J. Chem. Phys. 2010, 133 (14).

(20) Lim, S. H.; Bjorklund, T. G.; Spano, F. C.; Bardeen, C. J. Phys. Rev. Lett. 2004, 92, 107402.

(21) Burdett, J. J.; Bardeen, C. J. J. Am. Chem. Soc. 2012, 134, 8597. 
(22) Weiss, L. R. L. R.; Bayliss, S. L. S. L.; Kraffert, F.; Thorley, K. J. K. J.; Anthony, J. E. J. E.; Bittl, R.; Friend, R. H. R. H.; Rao, A.; Greenham, N. C. N. C.; Behrends, J. Nat. Phys. 2017, 13, 176.

(23) Stern, H. L.; Musser, A. J.; Gelinas, S.; Parkinson, P.; Herz, L. M.; Bruzek, M. J.; Anthony, J.; Friend, R. H.; Walker, B. J. Proc. Natl. Acad. Sci. U. S. A. 2015, 112,7656 .

(24) Stern, H. L.; Cheminal, A.; Yost, S. R.; Broch, K.; Bayliss, S. L.; Chen, K.; Tabachnyk, M.; Thorley, K.; Greenham, N.; Hodgkiss, J. M.; Anthony, J.; Head-Gordon, M.; Musser, A. J.; Rao, A.; Friend, R. H. Nat Chem 2017, doi 10.1038/nchem.2856.

(25) Arias, D. H.; Ryerson, J. L.; Cook, J. D.; Damrauer, N. H.; Johnson, J. C. Chem. Sci. 2016, 7, 1185.

(26) Ryerson, J. L.; Schrauben, J. N.; Ferguson, A. J.; Sahoo, S. C.; Naumov, P.; Havlas, Z.; Michl, J.; Nozik, A. J.; Johnson, J. C. J. Phys. Chem. C 2014, 118, 12121.

(27) Meyenburg, I.; Breuer, T.; Karthäuser, A.; Chatterjee, S.; Witte, G.; Heimbrodt, W. Phys. Chem. Chem. Phys. 2016, 18, 3825.

(28) Faltermeier, D.; Gompf, B.; Dressel, M.; Tripathi, A. K.; Pflaum, J. Phys. Rev. B 2006, 74, 125416 .

(29) Herz, J.; Buckup, T.; Paulus, F.; Engelhart, J. U.; Bunz, U. H. F.; Motzkus, M. J. Phys. Chem. A 2015, 119, 6602.

(30) Piland, G. B.; Bardeen, C. J. J. Phys. Chem. Lett. 2015, 6, 1841.

(31) Berkelbach, T. C.; Hybertsen, M. S.; Reichman, D. R. J. Chem. Phys. 2013, $138,114102$.

(32) Pensack, R. D.; Grieco, C.; Purdum, G. E.; Mazza, S. M.; Tilley, A. J.; Ostroumov, E. E.; Seferos, D. S.; Loo, Y.-L.; Asbury, J. B.; Anthony, J. E.; Scholes, G. D. Mater. Horiz. 2017.

(33) Yost, S. R.; Lee, J.; Wilson, M. W. B.; Wu, T.; Mcmahon, D. P.; Parkhurst, R. R.; Thompson, N. J.; Congreve, D. N.; Rao, A.; Johnson, K.; Sfeir, M. Y.; Bawendi, M.; Swager, T. M.; Friend, R. H.; Baldo, M. A.; Voorhis, T. Van. Nat. Chem. 2014, 6 (6), 492.

(34) Pensack, R. D.; Tilley, A. J.; Parkin, S. R.; Lee, T. S.; Payne, M. M.; Gao, D.; Jahnke, A. A.; Oblinsky, D. G.; Li, P.-F.; Anthony, J. E.; Seferos, D. S.; Scholes, G. D. J. Am. Chem. Soc. 2015, 137, 6790.

(35) Mastron, J. N.; Roberts, S. T.; McAnally, R. E.; Thompson, M. E.; Bradforth, S. E. J. Phys. Chem. B 2013, 117, 15519.

(36) Tayebjee, M. J. Y.; Schwarz, K. N.; MacQueen, R. W.; Dvořák, M.; Lam, A. W. C.; Ghiggino, K. P.; McCamey, D. R.; Schmidt, T. W.; Conibeer, G. J. J. Phys. Chem. C 2016, 120, 157.

(37) Odom, S. A.; Parkin, S. R.; Anthony, J. E. Org. Lett. 2003, 5, 4245.

(38) Kasai, H.; Oikawa, H.; Okada, S.; Nakanishi, H. Bull. Chem. Soc. Jpn. 1998, $71,2597$. 
(39) Kim, H. Y.; Bjorklund, T. G.; Lim, S.-H.; Bardeen, C. J. Langmuir 2003, 19, 3941.

(40) Akselrod, G. M.; Deotare, P. B.; Thompson, N. J.; Lee, J.; Tisdale, W. A.; Baldo, M. A.; Menon, V. M.; Bulović, V. Nat. Commun. 2014, 16 (5), 3646.

(41) Tayebjee, M. J. Y.; Clady, R. G. C. R.; Schmidt, T. W. Phys. Chem. Chem. Phys. 2013, 15 (35), 14797.

(42) Basel, B. S.; Zirzlmeier, J.; Hetzer, C.; Phelan, B. T.; Krzyaniak, M. D.; Reddy, S. R.; Coto, P. B.; Horwitz, N. E.; Young, R. M.; White, F. J.; Hampel, F.; Clark, T.; Thoss, M.; Tykwinski, R. R.; Wasielewski, M. R.; Guldi, D. M. Nat. Commun. 2017, 8, 15171.

(43) Chan, W.-L.; Ligges, M.; Zhu, X.-Y. Nat. Chem. 2012, 4 (10), 840.

(44) Bakulin, A. A.; Morgan, S. E.; Kehoe, T. B.; Wilson, M. W. B.; Chin, A. W.; Zigmantas, D.; Egorova, D.; Rao, A. Nat. Chem. 2016, 8, 16.

(45) Yong, C. K.; Musser, A. J.; Bayliss, S. L.; Lukman, S.; Tamura, H.; Bubnova, O.; Hallani, R. K.; Meneau, A.; Resel, R.; Maruyama, M.; Hotta, S.; Herz, L. M.; Beljonne, D.; Anthony, J. E.; Clark, J.; Sirringhaus, H. Nat. Commun. 2017, 8, 15953.

(46) Scholes, G. D. J. Phys. Chem. A 2015, 119 (51), 12699.

(47) Benk, H.; Sixl, H. Mol. Phys. 1981, 42 (4), 779. 


\section{Supplementary information}

\section{Methods}

Excitation dependent time- resolved photoluminescence measurements were recorded using an intensified charge coupled device (iCCD). An iCCD is a 2-dimensional multi-channel detector that measures the whole spectrum at once. With a time resolution of under $2 \mathrm{~ns}$, it can measure to a range of $1 \mathrm{~ms}$. Detection system in an iCCD set-up is comprised of a gate, an amplifier and a detector. Photons emitted from the sample are converted to electrons that are then subject to a pulsed electric field that sends the electrons to the detector. The electrons are sent to a micro-channel plate (MCP) for amplification and then converted back to photons at a phosphor layer and are read by the CCD. The iCCD set-up used for the experiments presented in this work were performed with the output of a home-built NOPA as the excitation source, similar to those used in

the transient absorption set-up. Excitation wavelength of $530 \mathrm{~nm}, 500 \mathrm{~nm}$ and $480 \mathrm{~nm}$ were chosen from the NOPA output by band-pass filters, and are sent to the sample at a repetition rate of $1 \mathrm{kHz}$. The collected $\mathrm{PL}$ is focussed onto the slits of a spectrometer (Shamrock 303i, Andor) coupled to an iCCD (iStar DH740, Andor).

\section{Characterizing crystallinity of nanoparticles using TEM and XRD}

TIPS-Tc nanoparticles formed by re-precipitation are approximately around $30 \mathrm{~nm}$ in size and have a very interesting morphology as seen from the TEM images in figure 1: a crystalline core and an amorphous exterior. However, to ascertain the crystallinity of the sample, X-ray diffraction techniques such as GIWAX was tried, but deemed unsuccessful due to the low concentration of the sample. Hence we resort to going back to the electron micrographs and analyzing the sharp interference fringes seen in the core, a mark of crystallinity. Fourier- transforming these fringes gives rise to dspacing values as it creates the diffraction image of the reciprocal lattice. These dspacing values were compared to those calculated from simulated XRD from the crystal data of TIPS-Tc. The peaks in XRD that matched with the d-spacing values obtained from TEM are shown in figure 2. This proves the presence of certain crystalline phases in the nanoparticle core. However, the same analysis was done in 
the exterior of the nanoparticle from the same TEM images and no diffraction image of the reciprocal lattice was obtained and confirms that the nanoparticles are amorphous on the outside.

\section{Influence of excitation energy in multiple formation pathways and yield of TT states}

The TRES-PL data from main figure $3 \mathrm{a}$ when analyzed by means of a genetic algorithm (GA) revealed the presence of two distinct species as shown in figure 4, when excited at $470 \mathrm{~nm}$. While the fast decaying singlet shows structured vibronic emission peaks, the later-forming broad, red-shifted emission is what is identified as the TT state in this study. The rise time of above $1 \mathrm{~ns}$ of this second species matches with the rise time of the SE (stimulated emission) at $630 \mathrm{~nm}$ in ps-TA data (main figure 5) when excited at $480 \mathrm{~nm}$ and this is further evidence on strengthening that the positive late-rising feature in ps-TA data at $480 \mathrm{~nm}$ excitation is indeed SE from the TT state.

Further to the excitation dependent ps- TA experiment where multiple pathways for TT formation comprising of emissive TT states selective to the excitation, excitation dependent time- resolved photoluminescence measurements were performed and are shown in figure 5. As the excitation wavelength increases from $530 \mathrm{~nm}$ to $500 \mathrm{~nm}$ and $480 \mathrm{~nm}$, singlet excitation starts getting dominated by the broader, red-shifted TT emission. As seen from figure 4, while the singlet spectrum at $530 \mathrm{~nm}$ excitation does not change in spectral shape from $2 \mathrm{~ns}$ to $20 \mathrm{~ns}$, at $500 \mathrm{~nm}$ and $480 \mathrm{~nm}$ excitation the initially dominant singlet emission gives way to a broad emission band around 620 $\mathrm{nm}$, which has been assigned to the TT state. The time resolution for this experiment was $2 \mathrm{~ns}$, however we see that singlet-like (550 nm emission) and TT-like (630 nm emission) have different kinetics at higher excitation energies, but are similar at 530 $\mathrm{nm}$. This supports the claim that excitation energy induces formation pathways to various TT states, within the same TT manifold. Added evidence from excitation dependent ps-TA on amorphous films of TIPS-Tc in figure 6 shows the emergence of the positive feature around $630 \mathrm{~nm}$ when excited at $480 \mathrm{~nm}$, but not at $532 \mathrm{~nm}$. Thus, the formation of multiple, even emissive TT states is not a phenomenon limited to nanoparticles alone, but may be generalized to films as well.

Fs-TA data with a broadband excitation at $530 \mathrm{~nm}$ shown in main text (figure 4) shows the presence of two distinct species from SVD analysis. From global analysis 
by GA, two spectral components were obtained and after a comparison for spectral similarities, were assigned as singlet and TT states. The resulting kinetics for these two components obtained from GA are shown in figure 8 where we see a rapid loss of singlet population and a similar rise of the TT population. While the singlet population lives longer, this initial decay and the correlated TT rise-time is a process that happens under $200 \mathrm{fs}$ which brings out one of the major findings of this work also complimenting the work done on TIPS-Tc films ${ }^{1}$, where vibrationally coherent formation of TT states was observed within 300 fs. The singlet lives longer with a lifetime of $18 \mathrm{ps,}$ which is due to the nature of broadband excitation exciting subensembles and thus forming a distribution of multiple TT states which are only producible by tuning excitation energy with a narrow band excitation pulse (like in the ps-TA experiment).

Global population analysis of the excitation energy dependent picosecond TA data in main figure 5a results in the deconvolution of singlet and TT spectrum along with the kinetics associated with it. When both the kinetic traces are normalized with respect to the singlet maximum, the TT kinetic constants may be used to understand trends in its yield with respect to the singlet. The value associated with TT yield with respect to the singlet is the highest when excited at $532 \mathrm{~nm}$ and the lowest when excited at 480 $\mathrm{nm}$. In order to verify this trend obtained from GA kinetics, we did a similar TT yield calculation directly from the raw data, by decay associated spectra (DAS) from singular value decomposition (SVD) of the raw data, giving rise to similar observations. Figure 6 shows the DAS obtained from ps- TA data when excited at 532 $\mathrm{nm}, 500 \mathrm{~nm}$ and $480 \mathrm{~nm}$. DAS show spectra associated with singlet decay, singlet to TT conversion and TT decay, giving rise to three spectra and their respective lifetimes. Singlet fission is a bimolecular process, where a molecule in $\mathrm{S}_{0}$ state collides with the $S_{1}$ state, to give rise to a mixed $S_{1}$-TT state, which then decays into the TT state, followed by decoupling of TT into free triplets. Thus, the lifetime of the initial state, say $[\mathrm{A}]_{0}$ is given as,

$$
t_{\frac{1}{2}}=\frac{1}{k[A]_{0}}
$$

, where $\mathrm{k}$ is the rate constant associated with the decay of species A. Assuming that the initial concentration of this species is fixed, the lifetimes of $\mathrm{S}_{1}$, TT or the mixed intermediate $\left(\mathrm{S}_{1}-\mathrm{TT}\right)$ states is indirectly proportional to the respective rate constants. 
Thus, trend in TT yield with respect to the singlet with varying excitation energies may be derived from the ratio between the lifetimes of the singlet decay (B) and that of singlet to TT conversion (A) as given in table 1. To compare the TT yield obtained from normalized kinetics from the GA with that calculated from DAS time constants, the value obtained by the latter all excitations were normalized by 0.7 , the highest yield of TT given by GA at $532 \mathrm{~nm}$. The normalized values of TT yield from DAS time constants show that yield of TT is higher when excited at lower energy. Interestingly, the normalized values between that obtained from GA and DAS are strikingly similar as well.

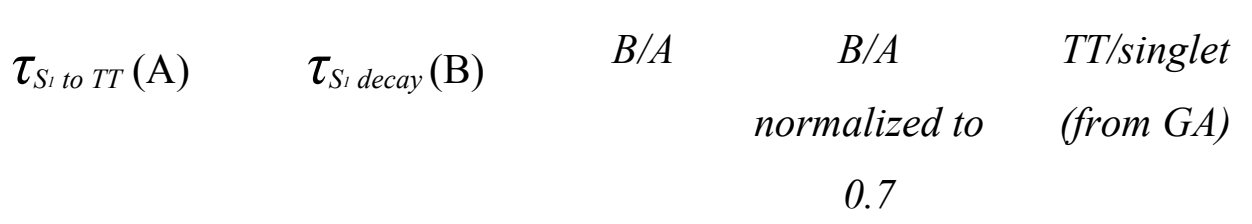

\begin{tabular}{|cccccc}
\hline 532 & $24 \mathrm{ps}$ & $2 \mathrm{ps}$ & 0.083 & $\mathbf{0 . 7}$ & $\mathbf{0 . 7}$ \\
500 & $57 \mathrm{ps}$ & $4 \mathrm{ps}$ & 0.070 & $\mathbf{0 . 5 9}$ & $\mathbf{0 . 4 8}$ \\
480 & $287 \mathrm{ps}$ & $14.3 \mathrm{ps}$ & 0.049 & $\mathbf{0 . 4 1}$ & $\mathbf{0 . 4}$ \\
\hline
\end{tabular}

Table 1. Compares TT to singlet conversion rate and singlet decay to estimate the yield of the TT state (B/A) with respect to the singlets, from rate constants obtained through DAS/SVD.

\section{Parallels between peak position shift and splitting of TT to form free uncorrelated triplets}

Figure 8a shows the time- dependent peak position change of this band along with kinetics at 830- $835 \mathrm{~nm}$ and $965-970 \mathrm{~nm}$ that are singlet and TT dominated kinetics respectively. The singlet- dominated kinetics $(830-835 \mathrm{~nm})$ has the earliest rise time, placing the singlet lifetime near $12 \mathrm{ps}$. The change in the peak position of the TT peak from 964- $972 \mathrm{~nm}$ differ from the singlet and TT rise observed for the 830- 835 and 965- 970 kinetics at early times before $1-10 \mathrm{~ns}$. The presence of large populations of singlets at picosecond timescales causes the initial rise time of the two to differ by one order of magnitude since the $964 \mathrm{~nm}$ peak was not spectrally resolved then. However, the kinetics match greatly irrespective of that. After $1 \mathrm{~ns}$, when there are no singlets left, TT dominated kinetics matches the change in TT peak position greatly and the longer second phase starting from $100 \mathrm{~ns}$ aligns with the formation of free triplets that 
are long- lived. The later ( $>$ 1ns) kinetics of the $830-835$ band follow the free triplet kinetics fairly, now it being a sharper triplet peak. Figure 10b shows a coherent picture of singlet fission in TIPS- Tc nanoparticles, the spectra obtained from the singlet, TT states, and triplets in the nanoparticles when excited at $532 \mathrm{~nm}$ with the timescales associated with them. The times associated with the early rise time of TT and the decay of singlet, decay of the TT state and slow formation of free triplets shown in complement each other.

\section{Morphology dependence in TT formation and triplet formation}

Figure 9 shows the TA spectra of TIPS- Tc films and solutions of various morphologies. The figure on top shows the TT signatures of disordered and polycrystalline films and $T_{1}$ signature of disordered films and sensitised solution of TIPS- Tc. While the major peak positions are the same at $835 \mathrm{~nm}$ and $968 \mathrm{~nm}$, there are significant differences in the orange region $880-950 \mathrm{~nm}$. The TT spectrum of polycrystalline films show a broad band at $910 \mathrm{~nm}$, but there are no free triplets reportedly forming in them. The TT spectrum of the disordered film shows features in this region, but within the noise limit and thus cannot be accounted for. The sensitised $\mathrm{T}_{1}$ spectrum of the solution $(200 \mathrm{mg} / \mathrm{mL})$ shows the presence of two narrow peaks at $905 \mathrm{~nm}$ and $925 \mathrm{~nm}$. Figure 10b shows the TT and $\mathrm{T}_{1}$ spectra of the nanoparticles assigned after comparison with existing data on films. The major peaks at $835 \mathrm{~nm}$ and $968 \mathrm{~nm}$ are similar to that observed in the disordered films, with $\mathrm{T}_{1}$ having sharper peaks. The presence of two sharp bands at $889 \mathrm{~nm}$ and $925 \mathrm{~nm}$, and a narrow band at $905 \mathrm{~nm}$ in the $T_{1}$ spectrum of the nanoparticles look very similar to the assignments of the solution sensitised spectrum. The difference between these sharp twin bands is $460 \mathrm{~cm}^{-1}$, which accords with the ground state Raman band at $470 \mathrm{~cm}^{-1}$ (C-C deformation vibration ${ }^{2}$ ) observed in TIPS- Tc films. The prompt TT state decays into sharper bands at $830,889,925 \mathrm{~nm}$ and is also linked with the shift in the position of the $964 \mathrm{~nm}$ peak to $972 \mathrm{~nm}$ from 1 ps to $1 \mu \mathrm{s}$, as it is a TT marker band, shown in figure 10a. 


\section{Supplementary figures}
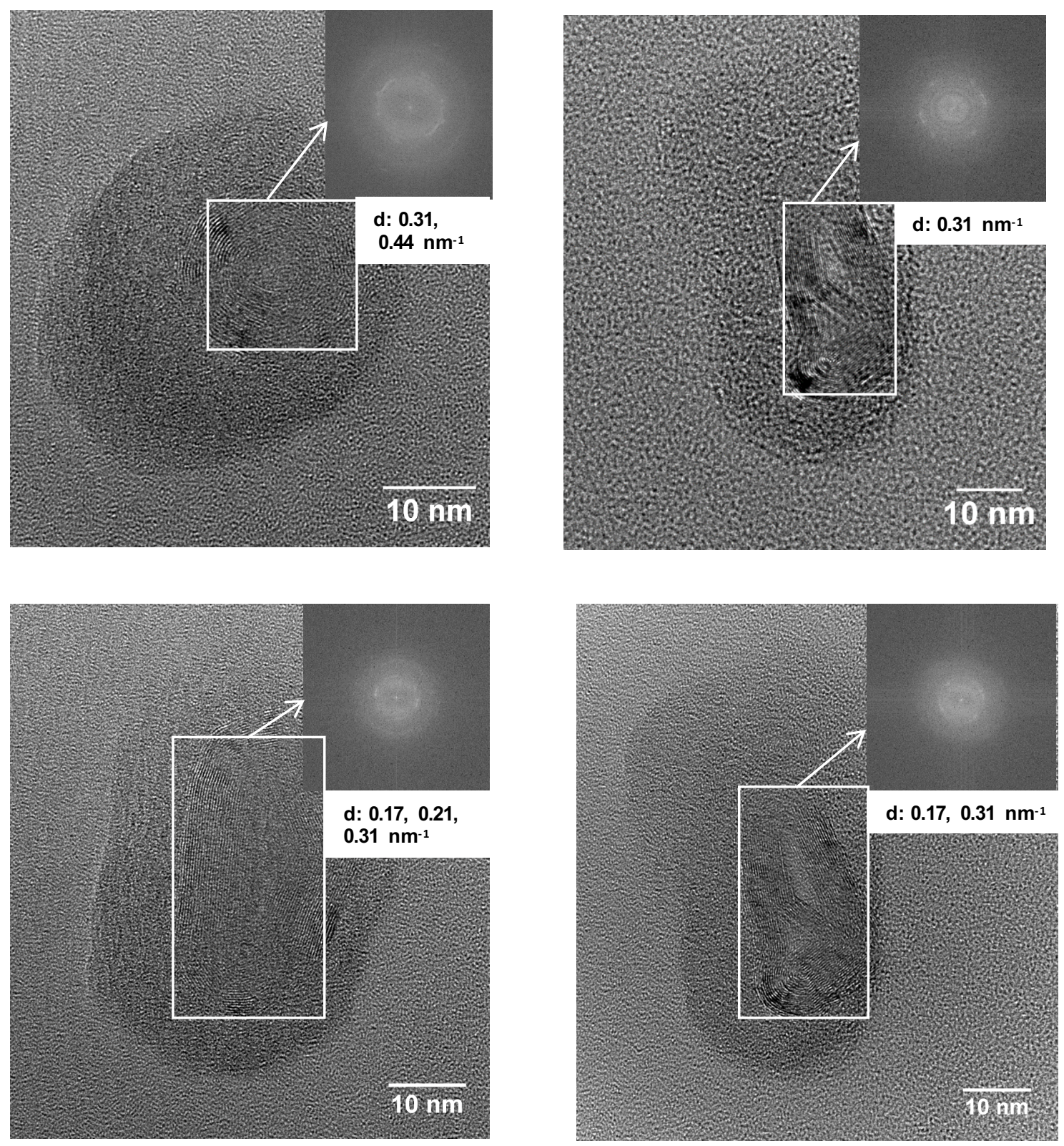

Figure 1. TEM images captured of samples made on different days. The size of all observed structures are similar $(\sim 30 \mathrm{~nm})$ and have surprisingly reproducible morphology of a crystalline core and an amorphous exterior. Diffraction patterns depicting reciprocal lattice of the crystalline core were obtained by Fourier transformation and have been included as insets in each individual figures. The dspacing values associated with them are also marked on the figures. 


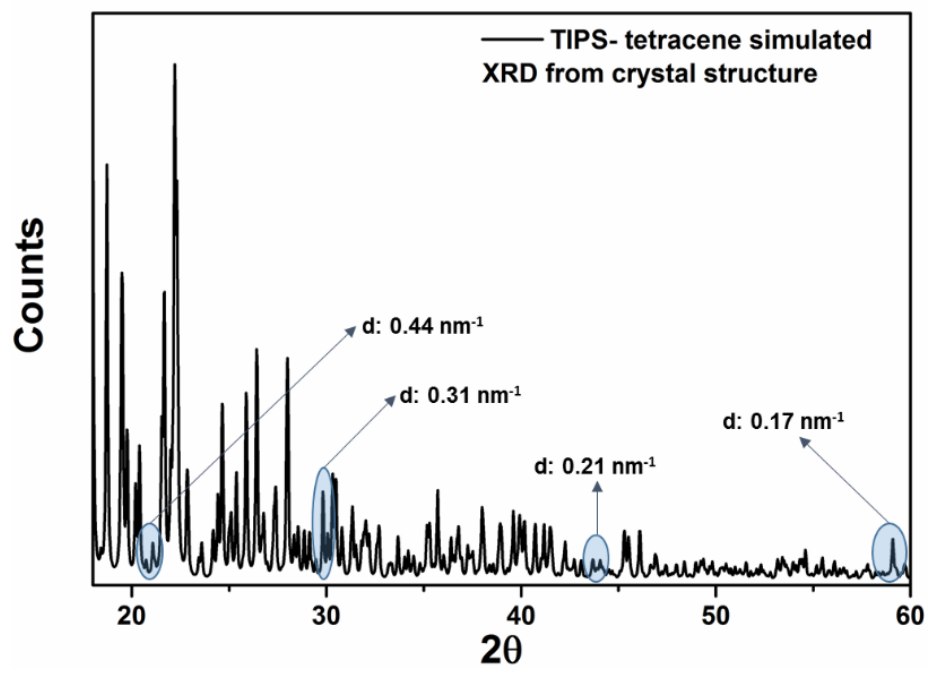

Figure 2. X-ray diffraction pattern simulated from its crystal structure. The d-spacing values from TEM FFT analysis was matched to some peaks in the XRD pattern and their $(\mathrm{h}, \mathrm{k}, \mathrm{l})$ were matched. This suggests some degree of crystallinity of the nanoparticle core.
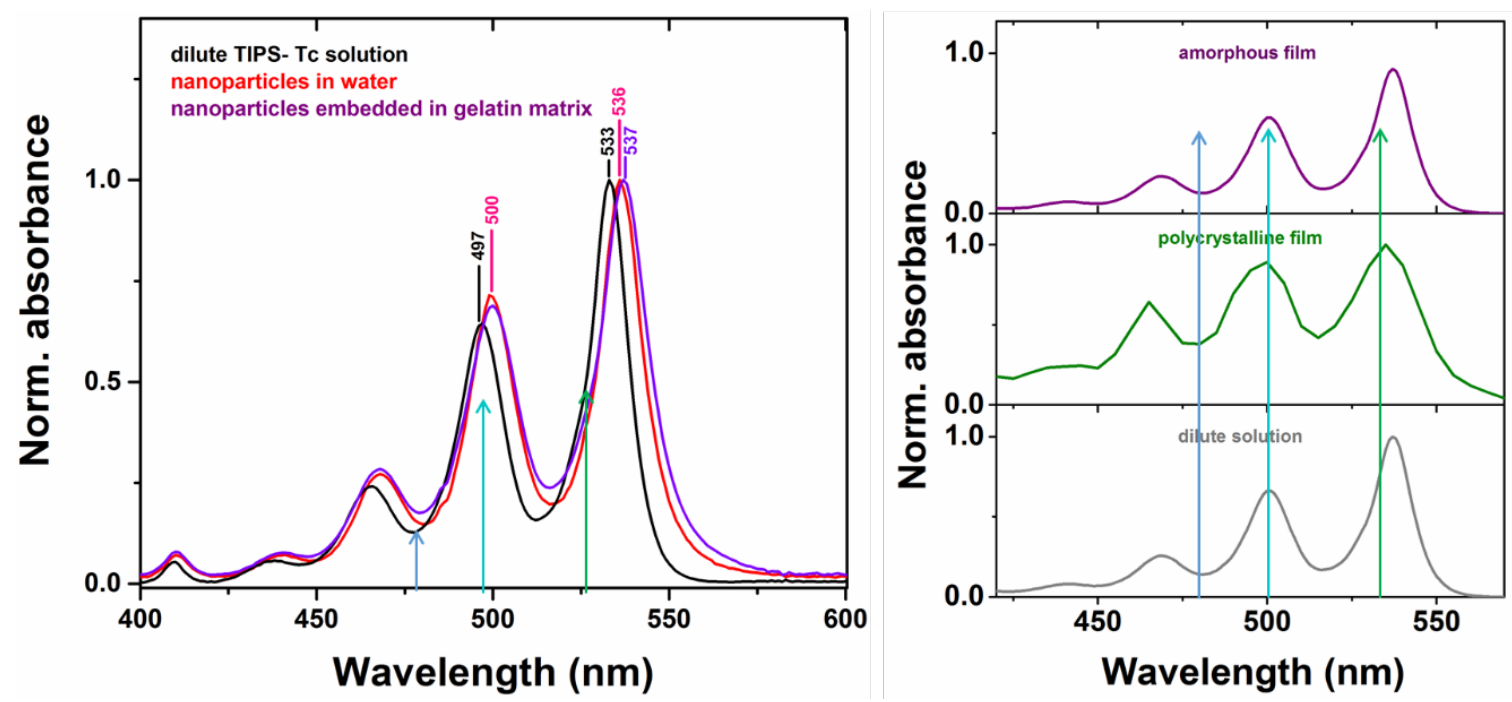

Figure 3. Absorption spectra of TIPS- Tc solution (black), nanoparticles in water (red) and nanoparticles in gelatin matrix (purple) in the left panel and that of amorphous film (purple), polycrystalline film (green) and dilute solution (grey) in the right panel. The slight change in absorption spectrum (shift of about $3 \mathrm{~nm}$ ) from molecular solution to the nanoparticles may suggest varying crystallinity, however absorbance at excitation wavelengths for other experiments presented in this work, $532 \mathrm{~nm}$ (green arrow), $500 \mathrm{~nm}$ (cyan arrow) and $480 \mathrm{~nm}$ (blue arrow), remain largely unchanged. The absorption spectra across different film morphologies also remain 
fairly unchanged, suggesting that there is no selective excitation of domains based on crystallinity at these energies.
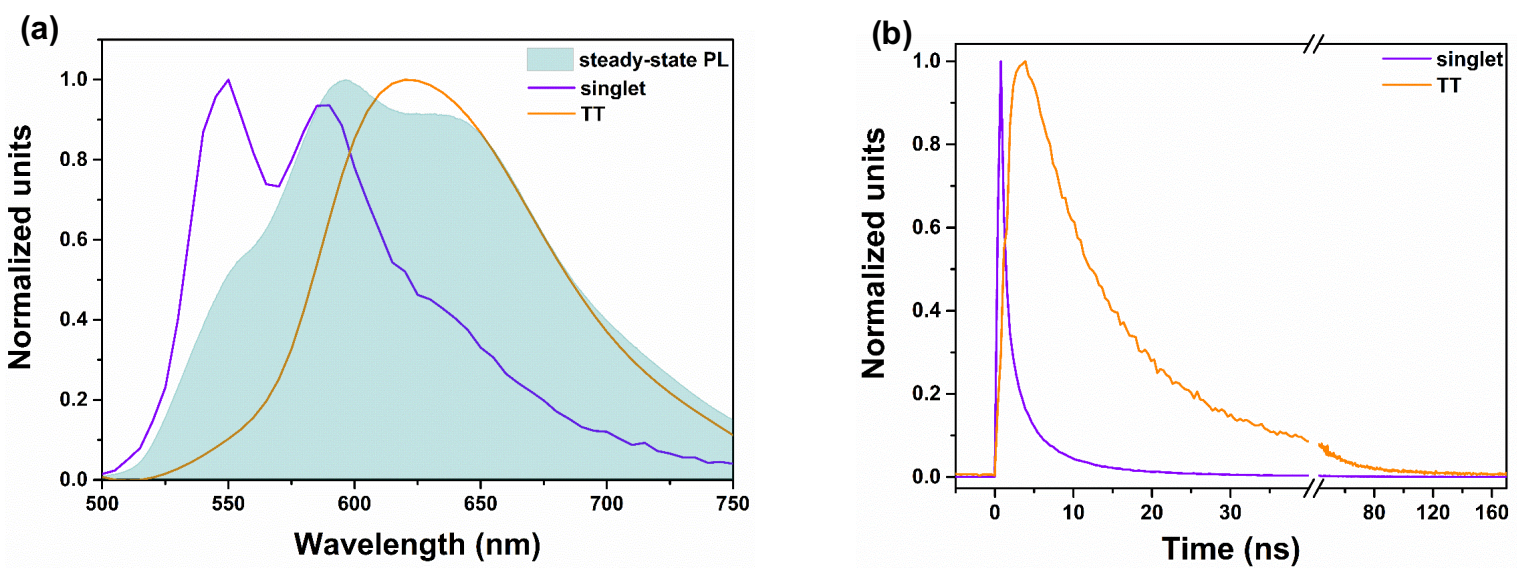

Figure 4. (a) GA spectral components 1 and 2 (2 components shown by SVD) analysis of TRES data shown in figure 3, when the nanoparticles were excited at 470 $\mathrm{nm}$. The components resemble singlet emission with an estimated time- constant within the instrumental limit $(<300 \mathrm{ps})$, and a later forming low- energy species with broad emission and a time- constant of above $20 \mathrm{~ns}$. The spectral components have been overlaid with the steady- state photoluminescence spectrum. (b) Kinetics of the two spectral components from GA show a fast- decaying singlet and a slow- decaying TT state with a rise time of over $1 \mathrm{~ns}$. This rise time of TT emission is consistent with the rise time of stimulated emission of the TT state around $630 \mathrm{~nm}$ in the ps TA data in figure $5 \mathrm{a}$.
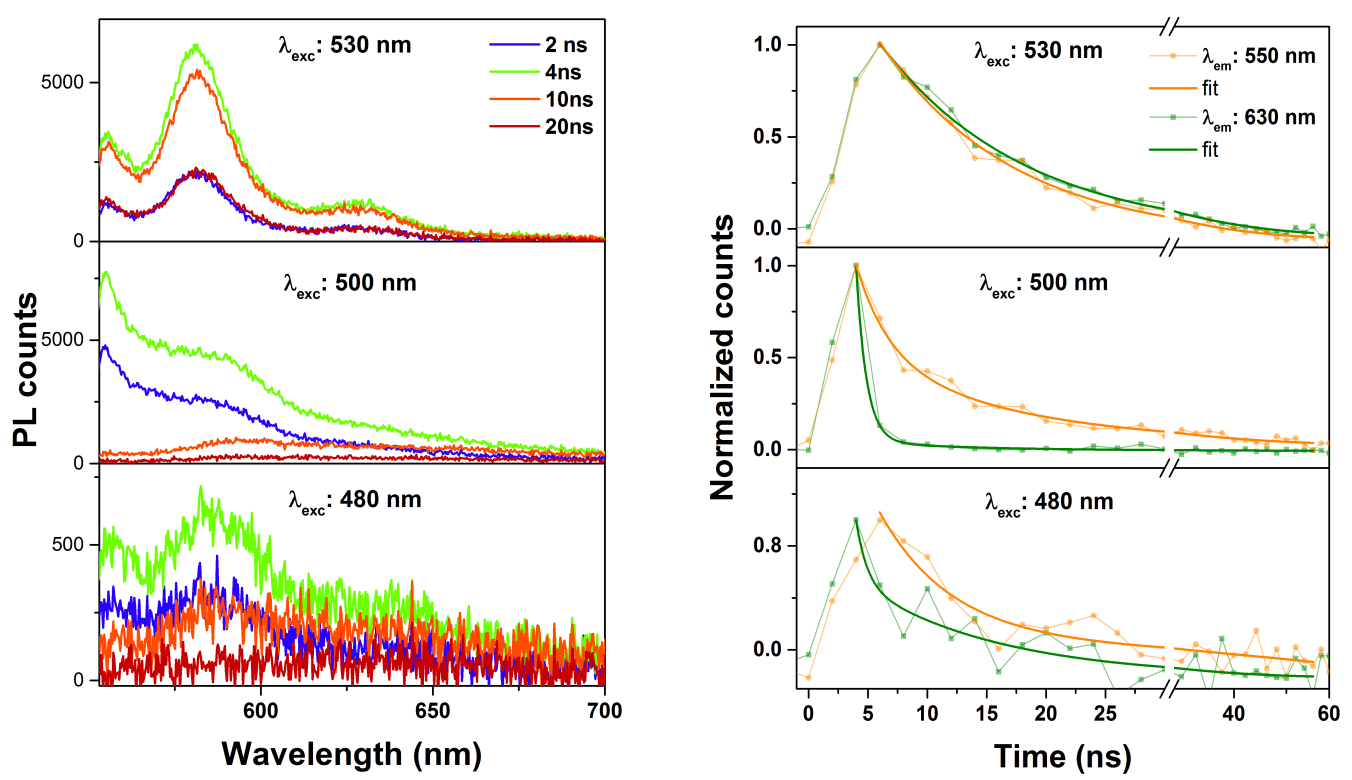
Figure 5. Time-resolved photoluminescence spectra with excitations at $530 \mathrm{~nm}, 500$ $\mathrm{nm}$ and $480 \mathrm{~nm}$. Spectra (left) show that when excited at $532 \mathrm{~nm}$ emission is dominated by the singlet and at higher energies emission is dominated by a redshifted broad emission. Kinetics (right) traces at $550 \mathrm{~nm}$ (singlet-dominated) and 630 $\mathrm{nm}$ (TT-dominated) show the differences in dynamics at different excitation energies. As the TT is more emissive at higher energies, the kinetics of this component clearly deviates form that of the singlet, unlike that at $530 \mathrm{~nm}$ excitation.



Figure 6. Decay associated spectra (DAS) calculated by using SVD from the picosecond transient absorption (ps-TA) experiment using narrowband excitations at $532 \mathrm{~nm}, 500 \mathrm{~nm}$ and $480 \mathrm{~nm}$ (data in main figure 5a). The three DAS species obtained from each experiment depict singlet decay (black), singlet to TT conversion (red) and TT decay (blue), with varying time constants. The ratio between time constants associated with singlet decay and singlet to TT conversion provides an understanding about TT yield as a function of excitation energy. 


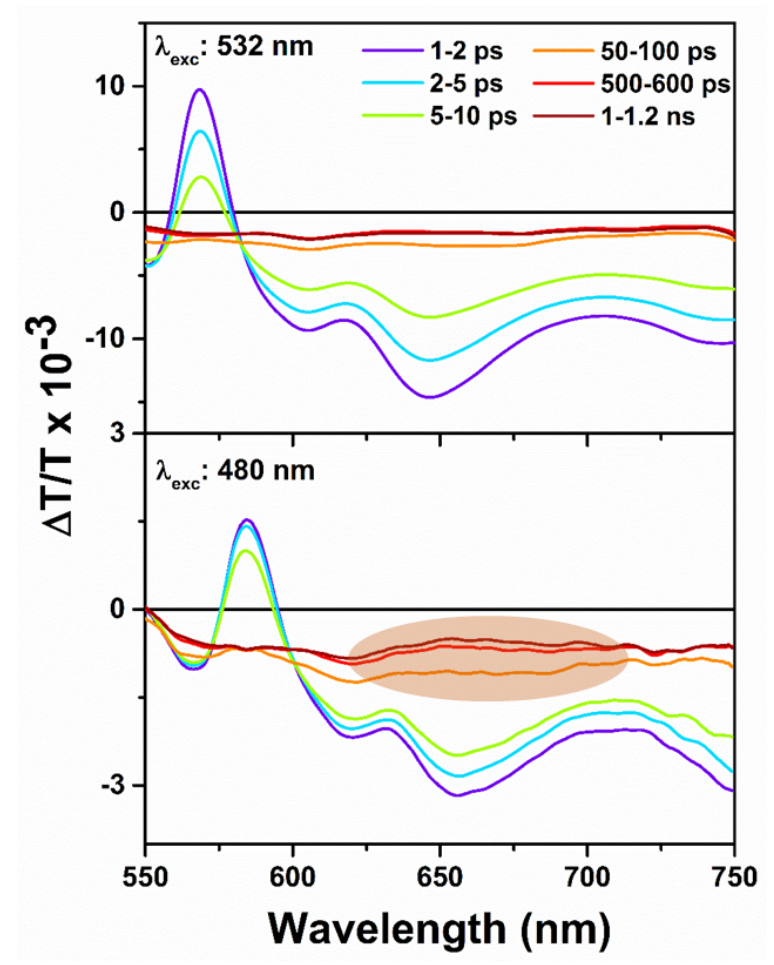

Figure 7. Picosecond transient absorption (ps-TA) experiment using narrowband excitations at $532 \mathrm{~nm}$ and $480 \mathrm{~nm}$ on an amorphous film of TIPS- Tc. Panel shows spectra at time delays integrated between 1-2 ps to 1-1.2 ns with visible probe ranging between 550 to $750 \mathrm{~nm}$. The initial forming singlet (SE at $580 \mathrm{~nm}$ and PIA at $650 \mathrm{~nm}$ ) decays into TT state (PIA at $610 \mathrm{~nm}$ ) at both excitation energies. Notice a positive feature growing in around $650 \mathrm{~nm}$ (orange highlight) above $100 \mathrm{ps}$ when excited at $480 \mathrm{~nm}$, but not at $532 \mathrm{~nm}$.

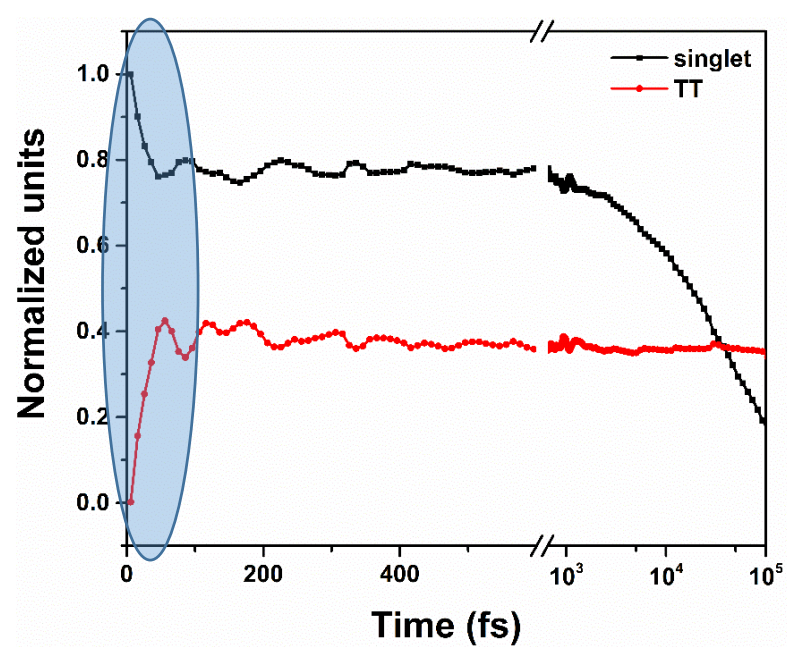

Figure 8. Kinetics of singlet and TT populations obtained by global analysis of fs-TA data shown in main figure 4 by a femtosecond, broadband excitation at $530 \mathrm{~nm}$. 
Corresponding components obtained from global analysis by means of a genetic algorithm (GA) are shown in main figure $4 \mathrm{~b}$. The kinetics show a rapid decay of the singlet population along with a prompt rise of the TT population within 200 fs. The singlet lives longer further with a lifetime of 18 ps and TT gradually decays with a nanosecond lifetime.

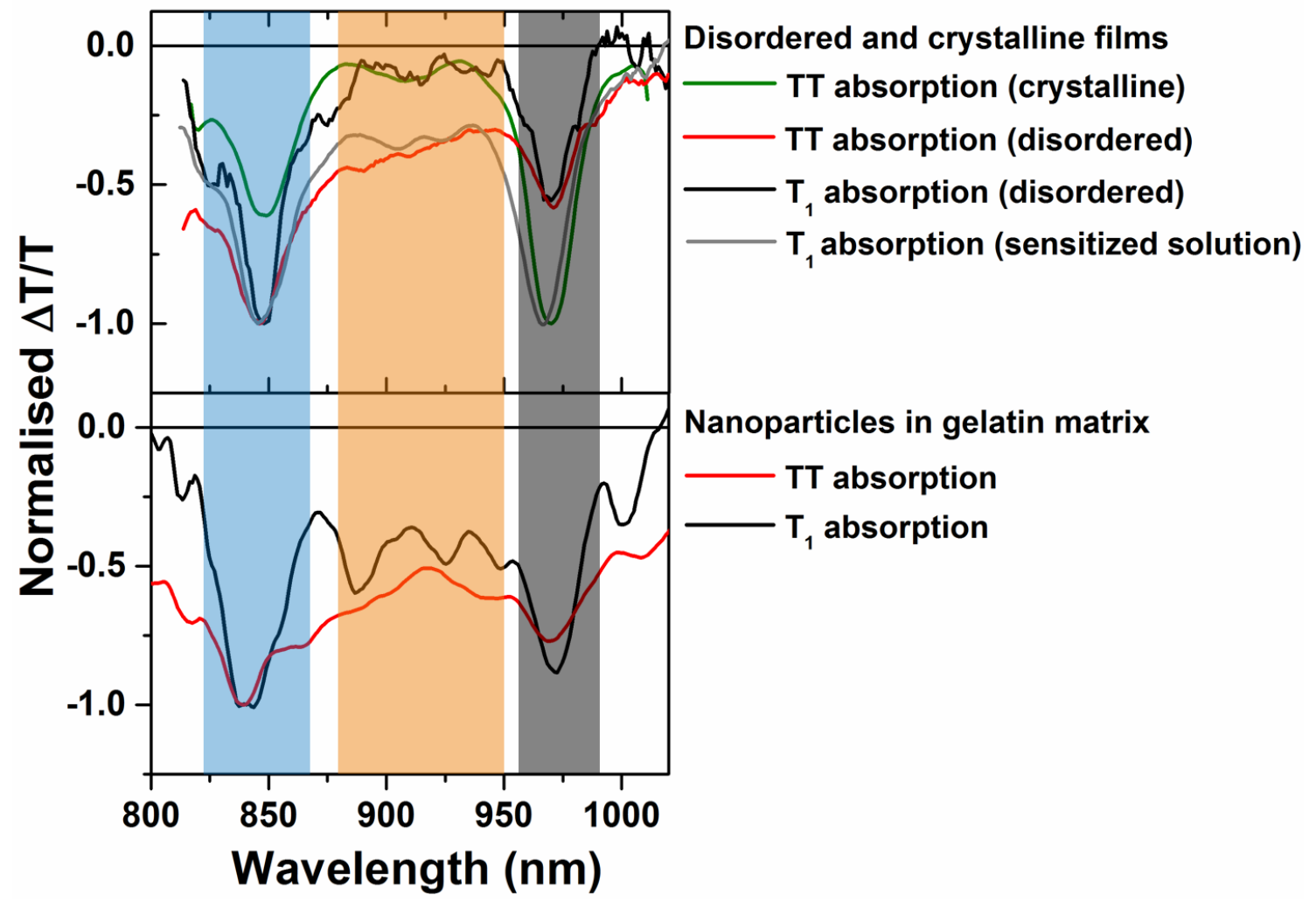

Figure 9. Transient absorption spectral signatures of $\mathrm{TT}$ and $\mathrm{T}_{1}$ absorption obtained in disordered films and nanoparticles and TT signature in crystalline films. The lower energy band at $848 \mathrm{~nm}$ has blue-shifted by $16 \mathrm{meV}$ in the nanoparticles compared to the disordered and crystalline films while the high energy band at $995 \mathrm{~nm}$ remains unaltered across all three samples in their TT/ $\mathrm{T}_{1}$ spectra. The broader band at $910 \mathrm{~nm}$ found in the TT spectra of the disordered and crystalline films has split into two small bands at $890 \mathrm{~nm}$ and $925 \mathrm{~nm}$ in the $\mathrm{T}_{1}$ spectrum of the nanoparticles. This splitting energy of $57 \mathrm{meV}$ corresponds to a ground state Raman band at $470 \mathrm{~cm}^{-1}$ in TIPStetracene. 

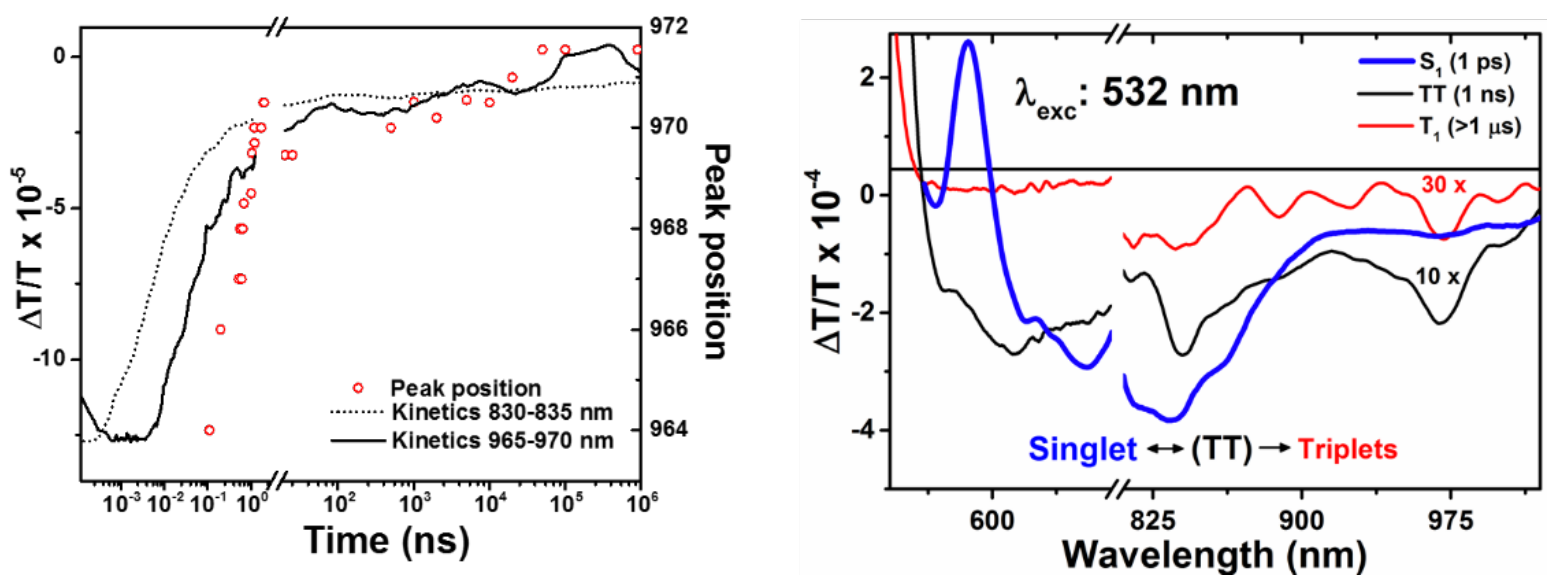

Figure 10. (a) Kinetics from the TA data when excited at $532 \mathrm{~nm}$ of largely singlet region in early times $830-835 \mathrm{~nm}$ (dotted black) and TT region 965-970 nm (solid black) which over tens of nanoseconds become a triplet band. Change in the position of the TT-> triplet peak between $964-972 \mathrm{~nm}$ (red circles) is plotted against time over the kinetics from TA, which exhibits a similar bi- phasic behaviour as the TT kinetics does. (b) DAS assigned to singlet (1 ps- blue), TT ( 1 ns- black) and triplet ( 1 us- red) TA spectral signatures in the nanoparticles when excited at $532 \mathrm{~nm}$. The times associated with the two- phase process of early- time rise in the TT state and the decay of singlets, and the slower formation of free triplets seen in (a) and (b) complement each other. 


\section{References}

(1) Stern, H. L.; Cheminal, A.; Yost, S. R.; Broch, K.; Bayliss, S. L.; Chen, K.; Tabachnyk, M.; Thorley, K.; Greenham, N.; Hodgkiss, J. M.; Anthony, J.; Head-Gordon, M.; Musser, A. J.; Rao, A.; Friend, R. H. Nat Chem 2017, doi 10.1038/nchem.2856.

(2) Alajtal, A. I.; Edwards, H. G. M.; Elbagerma, M. A.; Scowen, I. J. Spectrochim. Acta - Part A Mol. Biomol. Spectrosc. 2010, 76, 1. 